
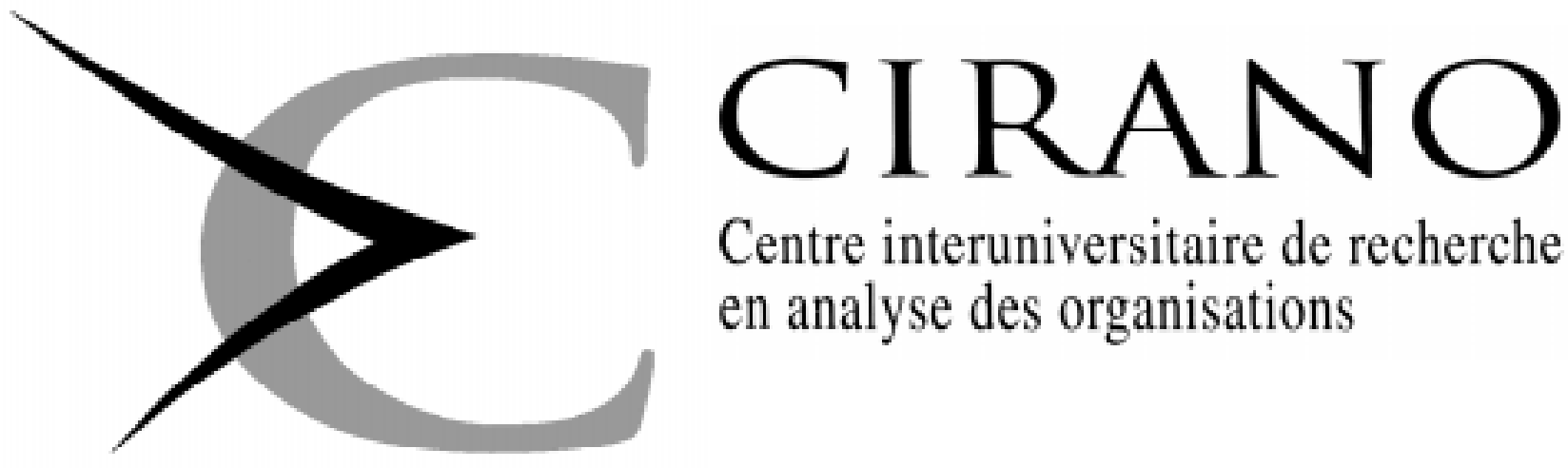

Centre interuniversitaire de recherche en analyse des organisations

Série Scientifique

Scientific Series

98s-41

\title{
Dynamic Equilibrium with \\ Liquidity Constraints
}

Jérôme Detemple, Angel Serrat 


\section{CIRANO}

Le CIRANO est un organisme sans but lucratif constitué en vertu de la Loi des compagnies du Québec. Le financement de son infrastructure et de ses activités de recherche provient des cotisations de ses organisations-membres, d'une subvention d'infrastructure du ministère de l'Industrie, du Commerce, de la Science et de la Technologie, de même que des subventions et mandats obtenus par ses équipes de recherche. La Série Scientifique est la réalisation d'une des missions que s'est données le CIRANO, soit de développer l'analyse scientifique des organisations et des comportements stratégiques.

CIRANO is a private non-profit organization incorporated under the Québec Companies Act. Its infrastructure and research activities are funded through fees paid by member organizations, an infrastructure grant from the Ministère de l'Industrie, du Commerce, de la Science et de la Technologie, and grants and research mandates obtained by its research teams. The Scientific Series fulfils one of the missions of CIRANO: to develop the scientific analysis of organizations and strategic behaviour.

\section{Les organisations-partenaires / The Partner Organizations}

-École des Hautes Études Commerciales

-École Polytechnique

- Université Concordia

- Université de Montréal

- Université du Québec à Montréal

- Université Laval

- Université McGill

-MEQ

- MICST

- Alcan Aluminium Ltée

- Banque Nationale du Canada

-Bell Canada

- Caisse de dépôt et placement du Québec

-Développement des ressources humaines Canada (DRHC)

- Egis

- Fédération des caisses populaires Desjardins de Montréal et de l'Ouest-du-Québec

-Hydro-Québec

- Imasco

- Industrie Canada

- Microcell Labs inc.

- Raymond Chabot Grant Thornton

- Téléglobe Canada

-Ville de Montréal

CC 1998 Jérôme Detemple et Angel Serrat. Tous droits réservés. All rights reserved.

Reproduction partielle permise avec citation du document source, incluant la notice (C).

Short sections may be quoted without explicit permission, provided that full credit, including $\odot$ notice, is given to the source.

Ce document est publié dans l'intention de rendre accessibles les résultats préliminaires de la recherche effectuée au CIRANO, afin de susciter des échanges et des suggestions. Les idées et les opinions émises sont sous l'unique responsabilité des auteurs, et ne représentent pas nécessairement les positions du CIRANO ou de ses partenaires.

This paper presents preliminary research carried out at CIRANO and aims to encourage discussion and comment. The observations and viewpoints expressed are the sole responsibility of the authors. They do not necessarily represent positions of CIRANO or its partners. 


\title{
Dynamic Equilibrium with Liquidity Constraints
}

\author{
Jérôme Detemple ${ }^{\dagger}$, Angel Serrat ${ }^{\ddagger}$
}

\section{Résumé / Abstract}

Cet article examine une économie intertemporelle avec contraintes de liquidité. Celles-ci empêchent la monétisation des revenus futurs et génèrent une incomplétude endogène des marchés financiers. En contraste avec la littérature récente sur les contraintes d'investissement, nos contraintes de liquidité émergent naturellement lorsque les agents peuvent déclarer faillite et ont un horizon fini. Un individu, dont la contrainte de liquidité sature, decide optimalement (i) de déférer sa consommation en période de jeunesse, mais (ii) de l'augmenter lorsque la contrainte est active. Le taux d'intérêt et les prix des actifs financiers d'équilibre sont caractérisés sous des conditions générales sur les préférences et les dotations. En présence de contraintes de liquidité le rendement de l'actif non risqué décroît. De plus le CAPM par rapport à la consommation est valide, même lorsque la structure de base du marché est incomplète. Lorsque l'aversion relative par rapport au risque est homogène et constante l'incomplétude du marché renforce l'effet des contraintes de liquidité et réduit encore davantage le rendement non risqué. Cependant, ni la contrainte d'incomplétude, ni celles de liquidité ne nous permettent d'expliquer le niveau empirique du ratio de Sharpe pour des valeurs raisonables du taux d'aversion au risque. D'autres contributions de cet article comprennent (i) une caractérisation nouvelle du problème de consommation-portefeuille d'un individu sous contrainte conduisant a une solution explicite, (ii) une approche constructive de détermination de l'équilibre, et (iii) une procédure numérique qui nous permet d'aborder les problèmes de calcul qui se posent dans ce contexte.

We consider an intertemporal economy with liquidity constrained and unconstrained individuals. A liquidity constraint prevents marketability of future income and thus endogenously generates market incompleteness. In contrast with the existing literature on portfolio constraints, our liquidity constraints arise naturally whenever agents may default and have a finite horizon. Liquidity constrained individuals optimally (i) postpone consumption in early age and (ii) experience permanent consumption increases whenever the constraint binds. The
\end{abstract}

\footnotetext{
* Corresponding Author: Jérôme Detemple, CIRANO, 2020 University Street, 25th floor, Montréal, Qc, Canada H3A 2A5 Tel: (514) 985-4028 Fax: (514) 985-4039 e-mail: detemplj@ cirano.umontreal.ca We would like to thank Pierluigi Balduzzi, George Constantinides, Jose Scheinkman and Lucie Teplà for helpful discussions. Earlier versions of this paper were presented at the 1996 AMS meetings at the Courant Institute at NYU, the 1996 meetings of the Society for Economic Dynamics and Control at ITAM and MIT Economics. This version was presented at Boston College, the University of Utah, CIRANO, the London Business School, Boston University, the University of Chicago, Université de Lausanne, the 1998 Summer Meeting of the Econometric Society at UQAM, the 1998 INFORMS meeting in Tel Aviv and the 1998 EFA meetings at INSEAD. We thank participants for their comments.

${ }^{\dagger}$ McGill University and CIRANO

* University of Chicago
} 


\begin{abstract}
equilibrium interest rate and asset prices are characterized under very general assumptions on preferences and endowment processes. In the presence of liquidity constraints, the cumulative interest return is reduced. In addition, the CCAPM holds, even when the basic market structure is incomplete. With homogeneous relative risk aversion market incompleteness reinforces the effect of liquidity constraints and further reduces the riskless return. However, we show that neither incompleteness nor liquidity constraints can explain the empirical magnitude of the Sharpe ratio for admissible levels of risk aversion, independently of preferences and endowment assumptions. Additional contributions of the paper include (i) a new characterization of the consumption-portfolio problem of constrained individuals leading to an explicit solution, (ii) a constructive approach to the determination of equilibrium, and (iii) a numerical procedure to handle the forward-backward path-dependent computational problem arising with a liquidity constraint.
\end{abstract}

Mots Clés : Équilibre, contraintes de liquidité, marchés incomplets, hétérogénéité, consommation, taux d'intérêt, prime de risque du marché, inégalité différentielle stochastique récursive, méthode numérique

Keywords : Equilibrium, liquidity constraints, incomplete markets, heterogeneity, consumption, interest rate, equity premium, backward stochastic differential inequality, numerical method 


\section{Introduction.}

Intertemporal economies with frictionless security markets have been extensively studied in financial economics. Early contributions by Merton (1973) and Breeden (1979) have identified the structure of equilibrium prices and interest rates in the context of Markovian economies. Extensions by Duffie and Huang (1985), Duffie (1986) and Duffie and Zame (1989) have considered complete markets with more general uncertainty structure and drawn the connection with Arrow-Debreu equilibria. Consumption and portfolio policies of agents are also well understood. Closed form solutions have been obtained for a class of Markovian models (Merton (1971)) and models with Brownian filtrations and complete markets (Cox and Huang (1989, 1991), Karatzas, Lehoczky and Shreve (1987)), while detailed characterizations are available when markets are incomplete (Karatzas, Lehoczky, Shreve and Xu (1991), He and Pearson (1991)) or in the presence of portfolio constraints (Cvitanic and Karatzas (1993), Cuoco (1997)). However, little is known about the impact on the consumption-portfolio policies and equilibrium of another type of constraints which impinges on the intertemporal allocation of risks of most investors in financial markets, namely liquidity constraints. These constraints introduce a form of market incompleteness which is markedly different from the situation created by the type of portfolio constraints considered in the literature so far.

A liquidity constraint arises as a result of the restriction to borrow against one's future income. Labor income is a typical example of an endowment stream that, in general, cannot be marketed due to moral hazard reasons or due to observability but non-verifiability of the idiosyncratic payment stream. However, unlike previous attempts which capture this situation by introducing ad-hoc limits on short-sales for specific assets (for example, Constantinides et. al (1997), Basak and Cuoco (1997)), we only constrain the value of the marketable assets of an individual to be nonnegative at all times. This is equivalent to the requirement that any short position has to be fully collateralized with marketable assets. While this constraint is automatically satisfied by placing a nonnegativity constraint on consumption when the endowment consists exclusively of an initial lump-sum of money, it is not satisfied when income is received throughout lifetime. Thus, unlike the complete markets case, whenever there are liquidity constraints the timing of labor income payments matters for consumption and portfolio choice.

Early results on the impact of similar wealth constraints on the consumption-portfolio problem were provided by Scheinkman and Weiss (1986), He and Pages (1991) and Hindy (1995). In independent work, a control problem similar to ours is posed as a stopping time problem in El Karoui and Jeanblanc-Picque (1997). Also, the consumption-portfolio problem studied in the macroeconomics literature on "buffer-stock" models arising from Deaton (1991), can be obtained as a special case of our formulation when agents are only allowed to trade a riskless asset. However, the nature and properties of the solution, characterized by the path-dependency of the consumption and portfolio choices, have not been identified in previous work. In addition, the analysis of the impact of liquidity constraints on equilibrium prices and allocations has not been considered in the literature either.

Our liquidity constraint formulation can be rationalized as follows: imagine an economy in which an agent can default at any time. Suppose that there is a costless technology available that tracks the credit history of individuals (a credit bureau). In addition, purchases and sales are anonymous but short sales are not. Short-sales can be collateralized but, for moral hazard reasons, only traded assets are acceptable as collateral (thus, an agent cannot pledge his future labor income as collateral). If an agent defaults (i.e. walks away from any short position in any traded asset), he is not be able to borrow (set up a short position) thereafter any more. ${ }^{1}$ Lastly, suppose also that all agents have a finite lifetime horizon T. Now, if an agent is short

\footnotetext{
${ }^{1}$ This is different from the analysis in Kehoe and Levine (1993) and Alvarez and Jermann (1997), in which if an agent defaults, he is thereafter not allowed to take both long and short positions in asset markets. In our framework, long positions cannot be prevented after default because trade in asset markets is anonymous.
} 
in some asset at $\mathrm{T}-1$, he will simply not settle his short position at period $\mathrm{T}$ (i.e. default is optimal at T). Thus, nobody would be willing to let him take a short position on any asset at T-1 unless he pledges enough collateral to cover this short position. At T-2, the agent knows that he will not be able to set up an uncollateralized short position at T-1 and therefore has no incentive to protect his credit history: he will optimally default at T-1 on any uncollateralized short position established at T-2. But anticipating that, other agents will then not allow him to establish an uncollateralized short position at T-2. By induction this argument holds at all times. As a result, any short position taken at any time must be fully collateralized with marketable assets, or equivalently liquid wealth must be nonnegative at all times (where by liquid wealth we understand the net value of the portfolio of traded assets of an individual).

More generally all cash flows that are under some form of control by agents enjoy limited marketability and are naturally subject to liquidity constraints. Thus, future inflows of idiosyncratic income cannot be part of liquid wealth until they are effectively cashed. Liquidity constraints will thus restrict the ability of agents to smoothe their consumption profile by placing a lower bound on the value of their liquid wealth. This form of constraint is then substantially distinct from incomplete markets or portfolio constraints examined in the earlier literature.

In this paper we solve the consumption-portfolio problem and derive equilibrium prices in the presence of liquidity constraints. Our approach to the consumption-portfolio problem is constructive. We first show that the problem can be approached by introducing a suitable correction to state prices which solves a forward-backward inequality, and then we provide a characterization of the solution in terms of a purely backward inequality. This reformulation is of interest since (i) the existence of a solution to the backward inequality can be demonstrated and (ii) the backward inequality lends itself easily to numerical computations. The consumption policy is then obtained in closed form in terms of the corrected state price density.

Our continuous time formulation of the problem has advantages that go beyond mathematical convenience. In a discrete time economy in which asset prices have unbounded support, agents could never take short positions in risky assets since the constraint has to be met at all times with probability one. This is overly restrictive since it prevents even the very wealthy individuals from engaging in any short-selling activity at all times. Such pathologies are avoided in a continuous time framework because liquid wealth is a continuous process. The liquidity constraint is thus truly endogenized in the sense that individuals are free to choose any portfolio they wish as long as they remain solvent. Thus, no ad-hoc constraints on portfolio choices are required to model non-traded labor income.

Consumption behavior in the presence of a liquidity constraint has a priori surprising and economically interesting properties. The analysis of the control problem shows that the liquidity constraint is in fact a direct constraint on the consumption profile of an individual as opposed to a constraint on his/her portfolio policy. The implications for equilibrium are therefore radically different from those of models with portfolio constraints. This constraint induces an individual to shift consumption from the present to the future, uniformly across states of nature. Thus, consumption is deferred in early age while the agent builds up his liquid wealth. At later stages of the lifecycle, the individual compensates by consuming more than in the standard frictionless model with identical financial market. The deferral of consumption in early age ensures that the liquidity constraint can be met at all subsequent times. The pattern of consumption deferral also exhibits interesting and albeit counterintuitive properties. The liquidity constraint is more likely to be binding when the individual experiences positive income shocks. At these times, the individual wishes to transfer resources to less favorable states of nature and thereby run liquidity deficits. Since the constraint precludes this it induces an increase in consumption. Furthermore since the marginal utility of accumulated wealth is lowered along all possible future trajectories of the economy this increase in consumption is permanent. Successive occurrences of a binding liquidity constraint will be accompanied by successive permanent increases in consumption. This 
reflects the fact that the liquidity constraint induces a segmentation of the state space that prevents the agent from equalizing marginal utilities to state-price ratios uniformly across states of nature.

We show that one can characterize equilibrium prices in our economy through a representative agent formulation even though the competitive equilibrium is not Pareto Optimal. With this characterization, we show that the equity premium satisfies the standard consumption CAPM. However, the liquidity constraint plays a role in the determination of expected returns since it affects the relative risk aversion parameter of the aggregator in the economy, which is a composite of the agents' risk aversions and depends on the distribution of consumption across agents. Since constrained individuals defer consumption in the early stages of life, the distribution of consumption is shifted toward unconstrained agents. The Sharpe ratio of the stock market unambiguously increases whenever aggregate risk aversion is a decreasing function of the consumption share of constrained individuals. We provide simple conditions under which this holds. Liquidity constraints, however, are unable to rationalize the empirical magnitude of the Sharpe ratio.

The cumulative equilibrium interest return bears the full direct impact of the constraint, while the risk premium of the stock reflects the constraint through the consumption allocation. In equilibrium, the cumulative interest return has two components. The first is a locally riskless interest rate $r$ which has the familiar structure: it is positively related to the expected consumption growth rate and negatively related to the aggregate consumption risk if the aggregator exhibits positive prudence. The second is a nonincreasing, singular component $A$ which is tied to the occurrence of a binding liquidity constraint. As mentioned above, whenever the constraint binds on an agent, his consumption demand experiences a permanent increase. Since the agent is prevented from borrowing, but not lending, at that time, the equilibrium interest rate must be lower than it would otherwise be. The yield to maturity on any short term bond will then have a tendency to decrease relative to an unconstrained economy. ${ }^{2}$ Accounting for liquidity constraints in asset pricing models may then help to resolve the riskfree rate puzzle (Weil (1990)).

Liquidity constraints have a similar impact on equilibrium when the basic market structure is incomplete. In such an environment the liquidity constraint reduces the riskless return and fails to impact the structure of the Sharpe ratio. Market incompleteness also has a direct effect on the riskless return but none on the structure of the Sharpe ratio. In economies populated by individuals with homogeneous constant relative risk aversion market incompleteness reinforces the effect of liquidity constraints and further reduces the riskless return. However, neither incompleteness nor liquidity constraints can be invoked to explain the magnitude of the Sharpe ratio.

The next section of the paper provides an intuitive example to motivate the relevance of liquidity constraints. Section 2 details the structure of the economy. Section 3 solves the consumptionportfolio problem in the presence of a liquidity constraint; the economic behavior of optimal consumption is analyzed and further intuitions are provided in the context of the example developed in the introduction. In section 4 we develop a constructive approach to the determination of equilibrium and investigate the properties of the interest rate and the equity premium. Section 5 extends some results to incomplete markets. All proofs are contained in the Appendix.

\subsection{An example.}

To illustrate the nature of the constraints that we investigate in this paper, we first solve an example for the consumption-portfolio problem of an unconstrained agent in a standard completemarkets framework. Consider the "binomial" tree economy with one stock and one bond and an investor with power preferences and a risk aversion coefficient equal to 3 . The investor is endowed only with a stream of labor income. The following lattice describes the evolution of the stock

\footnotetext{
${ }^{2}$ This holds if there is no offsetting effect due to a redistribution of consumption affecting the risk aversion of the aggregator. Constant and homogeneous relative risk aversion across agents is a sufficient condition for the unambiguous decrease in short term interest rates.
} 
price (which is denoted by $S$ ), the endowment of the agent (denoted by $e$ ), and the state-price density (denoted by $\xi$ ). Recall that the process $\xi$ makes $\xi_{t} S_{t}$ a martingale. Each time step in the lattice corresponds to one year. The implicit continuously-compounded interest rate is constant and equal to $5 \%$ per year. The probability of an up-movement in the lattice is constant and equal to 0.6545 . Time periods are $t=0,1,2$.

$$
\begin{aligned}
& \begin{array}{l}
e^{u u}=2.5495 \\
S^{u u}=1.5
\end{array} \quad \xi^{u u}=0.5488 \\
& e^{u}=1.5967 \\
& S^{u}=1.1937 \\
& \xi^{u}=0.7408 \\
& \begin{array}{l}
e=1 \\
S=1
\end{array}=1 \quad \searrow \\
& e^{d}=0.8496 \\
& S^{d}=0.9032 \\
& \xi^{d}=1.3499 \\
& \begin{array}{l}
e^{u d}=1.16 \\
S^{u d}=1
\end{array} \quad \xi^{u d}=1 \\
& e^{d d}=0.7219 \\
& S^{d d}=0.8970 \\
& \xi^{d d}=1.8222
\end{aligned}
$$

The continuous-time limit of this economy corresponds to a diffusion economy with lognormally distributed stock returns with Sharpe ratio equal to 0.3, and a continuously compounded interest rate of $5 \%$. The individual has a labor income stream whose expected growth rate is higher when he is young: the expected growth rate is $10 \%$ in period zero, and $2.45 \%$ on average in period one. In addition, the standard deviation of labor income is $30 \%$ in period zero, $37 \%$ in period one in the up-state and $23 \%$ in period one in the down-state.

In this economy, the investor attempts to maximize his lifetime utility of consumption

$$
E\left(\sum_{t=0}^{2} \frac{1}{1 \Leftrightarrow 3} c_{t}^{1-3}\right)
$$

by choosing a nonnegative consumption process $c$ and a trading strategy that specifies the number of shares of the stock to hold $(\Delta)$ and the number of one-period, $\$ 1$ face value zero-coupon bonds to hold $(B)$ at each node in the tree. The trading strategy is constrained to be self-financing in that

$$
\Delta_{t+1} S_{t}+B_{t+1}=\Delta_{t} S_{t}+B_{t} \exp (r)+e_{t} \Leftrightarrow c_{t}
$$

for $t=0,1$ with $\Delta_{0}=B_{0}=0$.

Since markets are complete and by standard arguments, rather than choosing $c, \Delta$ and $B$ that maximize (1) subject to (2), the agent can solve the consumption-portfolio choice problem just by choosing the consumption process $c$ that maximizes (1) subject to the single budget constraint:

$$
E\left(\sum_{t=0}^{2} \xi_{t} c_{t}\right)=E\left(\sum_{t=0}^{2} \xi_{t} e_{t}\right)
$$

The financing portfolio can then be obtained by recursive computation along the tree given the optimal consumption process.

The following tree shows the evolution of optimal consumption $(c)$, optimal stock and bond holdings at the end of each period, after retrade ( $\Delta$ and $B$ respectively) and the agent's wealth at the beginning of each period $(X)$. The Lagrange multiplier for the budget constraint $(3)$ is 0.5784 . 


$$
\begin{aligned}
& c^{u u}=1.4659 \\
& X^{u u}=\Leftrightarrow 1.0836 \\
& c^{u}=1.3264 \quad \Delta^{u}=\Leftrightarrow 2.2476 \\
& \begin{array}{lll}
X^{u}=\Leftrightarrow .7767 & B^{u}=2.2878 & \searrow \\
c^{d}=1.0860 & \Delta^{d}=\Leftrightarrow 2.1408 & \nearrow \\
X^{d}=0.3776 & B^{d}=2.181 & \searrow
\end{array} \\
& c=1.2002 \quad \Delta=\Leftrightarrow 3.9735 \\
& B=3.9665 \\
& c^{u d}=1.2002 \\
& X^{u d}=0.0402 \\
& c^{u d}=0.9826 \\
& X^{d d}=0.2607
\end{aligned}
$$

The portfolio of securities serves to finance future consumption in excess of the endowment (net consumption). At nodes $(u)$ and $(u u)$, the portfolio at the beginning of the period has a negative value: the agent is borrowing against his future labor income. For example, at the beginning of the third period at node $u u$, the individual owes 1.0836 , which is the value at the beginning of the third period of the portfolio that was chosen at the end of the second period. In the standard, default-free Arrow-Debreu setting, he would settle this debt at the end of the third period using the excess of his endowment (2.5495) over his consumption (1.4659) at that node.

Note, however, that the above program presumes that the individual never defaults on his debt. But, if income at node $u u$ cannot be fully appropriated by any creditor, the individual would prefer to default and consume 2.5495 rather than settle his short position and only consume 1.4659 . As a result his labor income will not be accepted as collateral by anyone who might consider taking the opposite side of his short portfolio position. It is then not possible for the individual to enter node $(u u)$ with negative liquidity in the first place, i.e. the anticipation of possible rational default precludes negative portfolio values. The same reasoning applies at prior nodes of the tree, in particular at node $u$ in period 2. Thus, we would like to solve the consumption-portfolio choice problem when liquid wealth $X$ is constrained to be nonnegative at all times. In other words we want to constrain the individual not to dispose of his labor income until it is cashed. This is in sharp contrast to the standard complete markets solution in which the individual is able to sell his lifetime labor income up-front if he wishes to do so. In section 3 we develop the solution procedure in the presence of such a liquidity constraint; we revert to the numerical example above in section 3.5 .

\section{The economy.}

We consider an intertemporal economy with finite horizon $[0, T]$ and two types of agents described below. The uncertainty is represented by a complete probability space $(\Omega, \mathcal{F})$, a probability measure $P$, a unidimensional Wiener process $W$ defined on $(\Omega, \mathcal{F})$ and a filtration $\mathcal{F}_{(\cdot)}$. We assume that $\mathcal{F}_{(\cdot)}$ is the natural filtration generated by $W$ and take $\mathcal{F}_{T}=\mathcal{F}$.

\subsection{Financial markets.}

A stock and a riskless asset are traded in financial markets (none of the results of this paper depends on the existence of a single stock). There is no outside supply of this asset, and there is only one share of the stock outstanding. The stock pays dividends $D$ such that

$$
d D_{t}=D_{t}\left[\mu_{t}^{D} d t+\sigma_{t}^{D} d W_{t}\right], \quad D_{0} \text { given }
$$

where $\mu^{D}$ and $\sigma^{D}$ are progressively measurable processes representing the drift and volatility of the dividend process. The stock price satisfies 


$$
\begin{aligned}
d S_{t}+D_{t} d t & =S_{t}\left[d m_{t}+\sigma_{t} d W_{t}\right] \\
d m_{t} & =\mu_{t} d t+d A_{t}
\end{aligned}
$$

subject to some initial value $S_{0}$, where $m$ and $\sigma$ are $\mathcal{F}_{(\cdot)}$-progressively measurable processes. The coefficient $m$ represents the cumulative drift of the stock price; it is composed of an instantaneous drift $\mu$ and a nonincreasing, singular process $A$ which is null at time 0 . As we will show later, the presence of liquidity constraints implies the emergence of such singular terms in equilibrium. The evolution of the riskless asset price $B$ is governed by the equation $d B_{t} / B_{t}=d R_{t}$ where

$$
d R_{t}=r_{t} d t+d A_{t}
$$

and where $r$ is an instantaneous rate of interest and $A$ is the singular component introduced above. This formulation is adopted from the start because we will prove later that the singular components in the stock and bond prices are necessarily identical in equilibrium: since our ultimate goal is to understand the structure of equilibrium we focus on the class of interest rate and stock price processes which are most relevant from that point of view. The initial value $S_{0}$, the coefficients $(m, \mu, A, \sigma)$, and the values of $B$ and $r$ are endogenous processes. To simplify notation, we define the discount factor

$$
b_{t}=\exp \left(\Leftrightarrow \int_{0}^{t} d R_{s}\right) .
$$

The value of a money market account, or a dollar continuously reinvested at the riskless spot interest rate, is $1 / b_{t}$. Taking the difference between the expected gross returns on the stock and the riskless asset and dividing by the stock price volatility gives the market price of risk $\theta=\sigma^{-1}(\mu \Leftrightarrow r)$. Though our economy presents incompleteness due to liquidity constraints, a unique equilibrium state price density will still exist. ${ }^{3}$ We will therefore maintain the following standard assumption which will enable us to solve the agents' consumption-portfolio problems.

Assumption 1: The market price of risk $\theta$ satisfies $E \exp \left(\frac{1}{2} \int_{0}^{T}\left\|\theta_{t}\right\|^{2} d t\right)<\infty$.

As we shall demonstrate this condition is satisfied in equilibrium under mild conditions: the class of relevant price processes is then the one satisfying (4), (6) along with Assumption 1. The corresponding state price density process (SPD) is

$$
\xi_{t}=b_{t} \exp \left(\Leftrightarrow \int_{0}^{t} \theta_{s} d W_{s} \Leftrightarrow \frac{1}{2} \int_{0}^{t}\left(\theta_{s}\right)^{2} d s\right) .
$$

and the Arrow-Debreu prices implicit in this market structure are $\xi_{t} d P$.

\subsection{Agents.}

The consumption space is the set of nonnegative, progressively measurable processes which satisfy the integrability condition, $\int_{0}^{T} c_{t}^{i} d t<\infty$, a.s., and such that utility (9) is finite. Agents have von Neuman-Morgenstern preferences defined on this space by

$$
U^{i}(c)=E\left(\int_{0}^{T} u^{i}\left(c_{t}, t\right) d t\right)
$$

\footnotetext{
${ }^{3}$ The market structure (4)-(6) is consistent with the absence of arbitrage opportunities (see Karatzas, Lehoczky and Shreve (1991)). An interpretation of the equilibrium state price density as the marginal utility of a representative agent in a Pareto-inefficient (constrained) equilibrium will be given below.
} 
$i=1, \ldots, I$. Let $u_{c}^{i} \equiv \partial u^{i} / \partial x$ denote the marginal utility of consumption. The following assumptions are standard.

Assumption 2: For each $i$ and for all $t \in[0, T]$

(i) $u^{i}(\cdot, t)$ is strictly increasing, strictly concave and twice continuously differentiable.

(ii) $\lim _{x \rightarrow 0} u_{c}^{i}(x, t)=\infty, \lim _{x \rightarrow \infty} u_{c}^{i}(x, t)=0$.

Each agent is endowed with an income process $e^{i}$ which is a nonnegative and integrable $\mathcal{F}_{(\cdot)}$-progressively measurable process. Initial endowments of shares of the stock are $n^{i}$ where $\sum_{i} n^{i}=1$. Initial endowments of the riskless asset are null.

Each agent chooses a consumption process $c^{i}$ and a portfolio process $\pi^{i}$ which represents his stock demand. The portfolio is a progressively measurable process which satisfies the integrability condition $\int_{0}^{T} \pi_{t}^{i}\left(\mu_{t} \Leftrightarrow r_{t}\right) d t+\int_{0}^{T}\left(\pi_{t}^{i} \sigma_{t}\right)^{2} d t<\infty, a . s$. . The admissible choice set for $c^{i}$ and $\pi^{i}$ will be specified below. The liquid wealth of an individual is the value of his portfolio of marketed assets: for a policy $\left(c^{i}, \pi^{i}\right)$ the liquid wealth process $X^{i}$ satisfies

$$
d X_{t}^{i}=X_{t}^{i} d R_{t}+\left(e_{t}^{i} \Leftrightarrow c_{t}^{i}\right) d t+\pi_{t}^{i}\left[\left(\mu_{t} \Leftrightarrow r_{t}\right) d t+\sigma_{t} d W_{t}\right]
$$

subject to the initial condition $X_{0}^{i}=n^{i} S_{0}$.

Individuals are divided into two categories depending on whether their income stream can be marketed or not. Recall that by "marketing" the labor income stream we mean using the future idiosyncratic income as collateral for today's aggregate short position. The set of all agents is $I=C \cup U$ where $C$ is the set of constrained agents (agents of type 1) and $U$ is the set of unconstrained agents (type 2). An example of an agent of type $U$ is an individual who inherits a claim to an exogenous production process (say, a firm); a typical individual of type $C$ is someone who only has a claim towards the income produced by his effort. The income of constrained agents $C$ must be either consumed or invested in marketed assets as it is obtained. Unconstrained agents can trade the stock and the riskless asset without restriction: markets are dynamically complete from their point of view. We naturally assume $C \cap U=\emptyset$.

A consumption-portfolio policy $\left(c^{i}, \pi^{i}\right)$ is admissible for agent $i$ if and only if the wealth process generated by $\left(c^{i}, \pi^{i}\right)$ satisfies

$$
\left\{\begin{array}{cll}
\xi_{t} X_{t}^{i}+E\left(\int_{t}^{T} \xi_{s} e_{s}^{i} d s \mid \mathcal{F}_{t}\right) \geq 0 & \forall t \in[0, T] & \text { if } \quad i \in U \\
X_{t}^{i} \geq 0 & \forall t \in[0, T] \quad \text { if } \quad i \in C
\end{array}\right.
$$

Let $\mathcal{A}_{i}$ be the admissible set for $i \in C \cup U$. Condition (11) states that a liquidity-constrained individual has to limit his consumption-portfolio choice to policies that ensure the nonnegativity of his liquid wealth (i.e. the value of his marketed assets) at all times and with probability one. On the other hand, an unconstrained individual can run temporary deficits in his liquid wealth position as long as his total wealth is nonnegative. Here total wealth equals liquid wealth plus the current value of his endowment stream. We can then say that the constrained individual faces a liquidity constraint while the unconstrained individual faces a solvency constraint. For later purposes, we shall record a useful property of the liquid wealth process generated by an admissible $\left(c^{i}, \pi^{i}\right)$.

Lemma 1 Let $\left(c^{i}, \pi^{i}\right) \in \mathcal{A}_{i}$ for $i \in C \cup U$. The liquid wealth process $X^{i}$ generated by $\left(c^{i}, \pi^{i}\right)$ satisfies the lower bound

$$
\xi_{t} X_{t}^{i} \geq E\left(\xi_{T} X_{T}^{i}+\int_{t}^{T} \xi_{s}\left(c_{s}^{i} \Leftrightarrow e_{s}^{i}\right) d s \mid \mathcal{F}_{t}\right)
$$

This condition says that liquid wealth corresponding to an admissible policy cannot be less than the present value of future net consumption where valuation is based on the SPD $\xi$. A pair $\left(c^{i}, \pi^{i}\right) \in \mathcal{A}_{i}$ is optimal for agent $i$ if and only if there is no other admissible pair $\left(\pi^{\prime}, c^{\prime}\right)$ such that $U^{i}\left(c^{\prime}\right)>U^{i}\left(c^{i}\right)$. 


\subsection{Definition of equilibrium.}

Let $\mathcal{E}$ denote the economy described above. A competitive equilibrium for $\mathcal{E}$ is a collection of stochastic processes $\left(\left(c^{i}, \pi^{i}\right)_{i \in C \cup U},(S, \mu, A, \sigma), r\right)$ such that (i) $\left(c^{i}, \pi^{i}\right)$ is optimal for $U^{i}, i \in C \cup U$, (ii) markets clear: (a) commodity market: $\sum_{i} c^{i}=\sum_{i} e^{i} \equiv e$, (b) stock market: $\sum_{i} \pi^{i}=S$, (c) riskless asset market: $\sum_{i}\left(\pi^{i} \Leftrightarrow X^{i}\right)=0$.

\section{Optimal consumption-portfolio policies.}

In this section we first describe an equivalent static consumption choice problem for a constrained agent (section 3.1). We then derive the optimal consumption policy (section 3.2), discuss consumption behavior (section 3.3), characterize the shadow price of the liquidity constraint (section 3.4) and provide numerical computation of the solution that allows and further economic intuition regarding the agent's lifetime consumption profile (section 3.5). To simplify notation we omit superscripts $i$ throughout the section.

\subsection{Static optimization with a liquidity constraint.}

It is well known that the dynamic consumption-portfolio problem of an unconstrained agent is related to a static problem involving consumption choice only (Cox and Huang (1989), Karatzas, Lehoczky and Shreve (1987)). We examine this relation in the presence of a liquidity constraint

The dynamic problem of a liquidity constrained individual is

$$
\sup _{(c, \pi)} U(c) \quad \text { s.t. } \quad(c, \pi) \in \mathcal{A}
$$

In order to formulate the static problem we recall that liquid wealth is bounded below by the present value of net consumption (12). Nonsatiation of preferences implies the following strenghtening of lemma 1

Lemma 2 If the utility function is strictly increasing liquid wealth satisfies

$$
\xi_{t} X_{t}^{i}=E\left(\int_{t}^{T} \xi_{s}\left(c_{s}^{i} \Leftrightarrow e_{s}^{i}\right) d s \mid \mathcal{F}_{t}\right)
$$

Consider then the static problem

$$
\begin{gathered}
\sup _{c} U(c) \text { s.t } \\
\left\{\begin{array}{l}
c \geq 0, \text { progressively measurable and integrable } \\
X_{t}=E\left(\int_{t}^{T} \xi_{s}\left(c_{s} \Leftrightarrow e_{s}\right) d s \mid \mathcal{F}_{t}\right) \geq 0 \quad t \in[0, T] \\
E\left(\int_{0}^{T} \xi_{t}\left(c_{t} \Leftrightarrow e_{t}\right) d t\right)=n S_{0}
\end{array}\right.
\end{gathered}
$$

The following proposition establishes the relationship between problems (13) and (15).

Proposition 3 Assume that $u$ satisfies the conditions of assumption 1. If $(c, \pi)$ solves the $d y$ namic problem (13), then $c$ solves the static problem (15)-(16). Conversely, if c solves the static problem (15)-(16), then there exists a portfolio policy $\pi$ such that $(c, \pi)$ solves the dynamic problem (13). Furthermore

$$
\pi_{t}=X_{t}\left(\sigma_{t}^{\prime}\right)^{-1} \theta_{t}+\xi_{t}^{-1}\left(\sigma_{t}^{\prime}\right)^{-1} \phi_{t}
$$

where $\phi$ is the unique, progressively measurable process in the representation of the martingale

$$
M_{t} \equiv E\left(\int_{0}^{T} \xi_{s}\left(c_{s} \Leftrightarrow e_{s}\right) d s \mid \mathcal{F}_{t}\right) \Leftrightarrow E\left(\int_{0}^{T} \xi_{s}\left(c_{s} \Leftrightarrow e_{s}\right) d s\right) .
$$




\subsection{The optimal consumption policy.}

The Lagrangian for the constrained static optimization problem is:

$$
\sup _{c} \inf _{y, \gamma} U(c)+y E\left(x \Leftrightarrow \int_{0}^{T} \xi_{t}\left(c_{t} \Leftrightarrow e_{t}\right) d t\right)+E\left(\int_{0}^{T} E\left(\int_{t}^{T} \xi_{s}\left(c_{s} \Leftrightarrow e_{s}\right) d s \mid \mathcal{F}_{t}\right) d \gamma_{t}\right)
$$

subject to $c \geq 0, y \geq 0$ and $d \gamma \geq 0$. Here $y$ is the multiplier for the static budget constraint and $d \gamma$ the multiplier for the liquidity constraint. Since the liquidity constraint must be satisfied at any time and in any state the multiplier $d \gamma$ is a stochastic process. Furthermore, $\gamma$ must be predictable and nondecreasing. This formulation is an application of optimization theory in infinite dimensional vector spaces (see details in Appendix B).

The Lagrangian (19) leads to the following first order conditions

$$
\left\{\begin{array}{l}
u_{c}\left(c_{t}, t\right)=\left(y \Leftrightarrow \gamma_{t}\right) \xi_{t} \\
E\left(\int_{0}^{T} \xi_{t}\left(c_{t} \Leftrightarrow e_{t}\right) d t\right)=n S_{0} \\
E\left(\int_{t}^{T} \xi_{s}\left(c_{s} \Leftrightarrow e_{s}\right) d s \mid \mathcal{F}_{t}\right) \geq 0 \\
E\left(\int_{0}^{T} X_{t} d \gamma_{t}\right)=0 \\
c_{t} \geq 0, y \geq 0 \\
\gamma \geq 0, \text { nondecreasing, null at } 0 .
\end{array}\right.
$$

The first equation states the usual equality of the marginal utility of consumption and of the state price density, accounting for the shadow prices of the constraints. The next two conditions correspond to the budget and liquidity constraints, respectively. The fourth condition is the complementary slackness condition: when the constraint is slack $\left(X_{t}>0\right)$ the multiplier is null $\left(d \gamma_{t}=0\right)$; when the multiplier charges $\left(d \gamma_{t}>0\right)$ the constraint is binding $\left(X_{t}=0\right)$. The last two sets of conditions are nonnegativity constraints on the control variables.

Our next result is a verification theorem which shows that the conditions (20) above are sufficient for the static optimization problem.

Theorem 4 Suppose that there exist progressively measurable processes $(c, \gamma)$ and a constant $y$ such that $(c, \gamma, y)$ solves (20). Then c solves (15) subject to (16).

A consequence of Theorem 4 is that we can focus on the conditions (20) to solve the static problem and identify the optimal consumption policy. This task is carried out in section $3.4{ }^{4}$

Remark 1 The first order conditions (20) also reveal that the optimal consumption of the constrained agent coincides with the consumption plan that he would choose in an artificial "shadow" economy without liquidity constraint, but in which the state price density is given by $(y \Leftrightarrow \gamma) \xi$ rather than $\xi$. Since $\gamma_{t}$ is a nondecreasing process, this "shadow" economy can be interpreted as the original economy but with a new riskfree return that experiences increases at selected times. In order to get the same consumption and wealth path in this artificial unconstrained economy, the riskfree return has to increase just enough to discourage the agent to run uncovered short

\footnotetext{
${ }^{4}$ Results on the existence of a solution (though not a constructive derivation of the solution) appear in Pagès (1989). He shows that an optimum to (15)-(16) exists if (i) the utility function is bounded, or if (ii) it is positive and there exists constants $a, b>0,0<p<1$ such that $u(x, t) \leq a+b x^{p}$, or (iii) it is unbounded and there exist constants $a, a^{\prime}, b>0, b^{\prime}>0, p<1, p^{\prime}<0$ such that $a^{\prime}-b^{\prime} x^{p^{\prime}} \leq u(x, T) \leq u(x, 0) \leq a+b x^{p}$.

Note that these sufficient conditions for existence are satisfied by power utility functions with a relative risk aversion different from one. The constructive derivation of the optimal policy that we carry out in section 3.4 does not require such restrictions on utility functions.
} 
positions at times when he would otherwise like to do so. Note however, that this reinterpretation of the model might be misleading: as we show later the liquidity constraint will actually lower the equilibrium riskfree return relative to an economy without liquidity constraint.

\subsection{Consumption behavior with a liquidity constraint.}

As the previous example shows, a wealth constrained individual exhibits a consumption behavior which departs significantly from the standard unconstrained case. Let $I(y, t)=\inf \{x \geq 0$ : $\left.u_{c}(x, t) \leq y\right\}$ denote the left-inverse of the marginal utility of consumption. The first order condition in (20) yields the optimal consumption policy

$$
c_{t}=I\left(\left(y \Leftrightarrow \gamma_{t}\right) \xi_{t}\right) .
$$

Risk aversion implies that the inverse marginal utility $I(\cdot)$ is strictly decreasing. Since $\gamma$ is a nondecreasing process, we conclude that the multiplier $y$ which solves the static budget constraint

$$
E\left(\int_{0}^{T} \xi_{t}\left(I\left(\left(y \Leftrightarrow \gamma_{t}\right) \xi_{t}\right) \Leftrightarrow e_{t}\right) d t\right)=n S_{0}
$$

exceeds the multiplier which would prevail in the absence of a wealth constraint. As explained earlier, the process $\gamma$ captures the wedge between the marginal rate of substitution between two time periods and the ratio of the state-prices corresponding to these states. The former will be smaller than the latter since the agent cannot borrow. The fact that $\gamma$ only increases at times when the constraint binds also means that the multiplier $\gamma$ reflects the anticipation of occurrence of future constrained states. Since the process $\gamma$ is null at zero, we also deduce that the agent reduces consumption in early age while increasing it at later stages in his life: early age corresponds to a period of buffer wealth buildup. The anticipation of future constrained states is the source of his conservative behavior at the outset. This optimal policy is radically different from the "naive" intuition suggesting that the individual starts off his consumption policy at levels more or less equal to the ones in the unconstrained case, and then readjusts his consumption downward when "bad times" occur in order to accumulate income, build up wealth and loosen the constraint.

Another striking difference with the standard consumption behavior is the presence of a singular component in the evolution of the consumption function. Indeed, applying Ito's lemma to the optimal consumption process gives

$$
\frac{d c_{t}}{c_{t}}=\frac{1}{R_{t}^{R}}\left[\left(\frac{P_{t}}{R_{t}^{R}}\right) \theta_{t}^{2}+\left(\frac{u_{t}}{u_{c}}\right)\right] d t+\frac{1}{R_{t}^{R}}\left(d R_{t}+\left(y \Leftrightarrow \gamma_{t}\right)^{-1} d \gamma_{t}\right) \Leftrightarrow \frac{1}{R_{t}^{R}} \theta_{t} d z_{t}
$$

where $R^{R}=\Leftrightarrow \frac{c u_{c c}}{u_{c}}$ denotes the relative risk aversion coefficient and $P=\Leftrightarrow \frac{c u_{c c c}}{u_{c c}}$ is the relative prudence coefficient. The volatility of consumption has the same structure as in the standard model. The drift coefficient, however, has the additional term $\left(R^{R}\right)^{-1}\left(y \Leftrightarrow \gamma_{t}\right)^{-1} d \gamma_{t}$ relative to the unconstrained case. This term reflects the impact of the wealth constraint. In states of nature in which the constraint is lax, we have $d \gamma=0$ and the consumption growth rate has the standard local behavior (in particular, the expected growth rate is absolutely continuous with respect to Lebesgue measure). But consumption does not grow at the same rate as in the unconstrained case since the consumption level anticipates future constrained states. Prior to reaching a constrained state the agent builds up his wealth by reducing consumption. Furthermore in those good states of nature in which the individual would like to deplete his wealth in order to improve his resources at other less favorable times, he can only do so to a limited extent since he cannot take aggregate short positions. Thus, each time the constraint binds, the agent has to readjust his consumption behavior upwards given his inability to cross-subsidize less favorable states. These intuitions will become clear below when we solve the consumption-portfolio problem in the tree example set up in the introduction. However, before proceeding to this example we first study the solution of the control problem in detail: this will enable us to develop an algorithm to compute the multiplier $\gamma$ and the optimal consumption-portfolio policy. 


\subsection{The shadow price of liquidity.}

In order to compute the consumption policy and shed further light on consumption behavior we need to compute the multiplier $\gamma$. In this section, we provide a characterization of $\gamma$ which facilitates its computation. The key to this characterization is the transformation of the forwardbackward structure of the original problem (20) into a purely backward structure. This is achieved by a change of variables.

To solve the control problem of a constrained investor we must identify a process $\gamma$ and a constant $y$ such that

$$
\left\{\begin{array}{l}
\xi_{t} X_{t}=E\left(\int_{t}^{T} \xi_{s}\left[I\left(\left(y \Leftrightarrow \gamma_{s}\right) \xi_{s}\right) \Leftrightarrow e_{s}\right] d s \mid \mathcal{F}_{t}\right) \geq 0, \quad t \in[0, T] \\
X_{t} d \gamma_{t}=0 \text { for all } t \in[0, T], \text { and } \gamma \text { nondecreasing null at } 0 \\
E \int_{0}^{T} \xi_{s}\left[I\left(\left(y \Leftrightarrow \gamma_{s}\right) \xi_{s}\right) \Leftrightarrow e_{s}\right] d s=n S_{0} .
\end{array}\right.
$$

For any adapted process $\Delta$ define the map

$$
\begin{gathered}
F_{t}\left(y, \Delta_{t}\right) \equiv E\left(\int_{t}^{T} \xi_{s}\left[I\left(\left(y \Leftrightarrow \Delta_{t, s}^{*}\right) \xi_{s}\right) \Leftrightarrow e_{s}\right] d s \mid \mathcal{F}_{t}\right) \geq 0 \\
\Delta_{t, s}^{*} \equiv \sup _{v \in[t, s]} \Delta_{v} .
\end{gathered}
$$

for $t \in[0, T]$. Consider the auxiliary problem: find an adapted process $\Delta$ and a constant $y$ such that

$$
\begin{gathered}
F_{t}\left(y, \Delta_{t}\right) \geq 0, \quad \forall t \in[0, T] \\
F_{t} d \Delta_{t} \leq 0 \text { for all } t \in[0, T], \text { and } \Delta \text { nonnegative null at } T_{+} \\
E \int_{0}^{T} \xi_{s}\left[I\left(\left(y \Leftrightarrow \Delta_{0, s}^{*}\right) \xi_{s}\right) \Leftrightarrow e_{s}\right] d s=n S_{0}
\end{gathered}
$$

Note that the first two conditions (23)-(24) in the auxiliary problem have a backward structure as opposed to the forward-backward structure of the original constrained problem. A closer look at the two problems also reveals that the process $\gamma$ in the constrained problem is given by

$$
\gamma_{t}=\Delta_{0, t}^{*}
$$

where $\Delta$ solves the backward problem. The auxiliary problem (23)-(25) then computes the "push" at date $t$ assuming that the constraint did not bind prior to $t$. This formulation is of interest because it provides a useful and simple characterization of the Lagrange multiplier process.

Lemma 5 Consider the backward problem (23)-(25). A solution is given by

$$
\Delta_{t}\left(y^{*}\right)=F_{t}^{-1}\left(y^{*}, 0\right) 1_{\left\{F_{t}\left(y^{*}, 0\right)<0\right\}}
$$

where $F_{t}^{-1}(y, 0)$ is the inverse of the map $F_{t}(y, \cdot)$, defined in (21), and the constant $y^{*}$ uniquely solves

$$
E \int_{0}^{T} \xi_{s}\left[I\left(\left(y \Leftrightarrow \Delta_{0, s}^{*}(y)\right) \xi_{s}\right) \Leftrightarrow e_{s}\right] d s=n S_{0}
$$

subject to $\Delta_{0}=0$.

The solution of the consumption problem of a liquidity-constrained agent now follows. 
Theorem 6 Define $\Delta_{v}(y)$ and $y^{*}$ as in (26)-(27) and consider the process $\gamma_{t}^{*}=\Delta_{0, t}^{*}\left(y^{*}\right) \equiv$ $\sup _{v \in[0, t]} \Delta_{v}\left(y^{*}\right)$. The optimal consumption policy of the constrained agent is given by

$$
c_{t}=I\left(\left(y^{*} \Leftrightarrow \gamma_{t}^{*}\right) \xi_{t}\right)
$$

and his optimal liquid wealth is $X_{t}=\left(\xi_{t}\right)^{-1} E\left(\int_{t}^{T} \xi_{s}\left[I\left(\left(y^{*} \Leftrightarrow \gamma_{s}^{*}\right) \xi_{s}\right) \Leftrightarrow e_{s}\right] d s \mid \mathcal{F}_{t}\right)$.

The characterization of the multiplier in Theorem 6 is relatively easy to implement numerically. The main idea underlying the result is to start the problem anew at each point in time (i.e. ignoring past occurrences of a binding constraint): this reduces the forward-backward equations satisfied by the multiplier $\gamma$ in the first-order conditions to a purely backward equation.

Given the optimal consumption policy in Theorem 6 and the results in Proposition 3 we obtain the following characterization of the optimal portfolio.

Lemma 7 The optimal portfolio policy is $\pi_{t}=X_{t}\left(\sigma_{t}^{\prime}\right)^{-1} \theta_{t}+\xi_{t}^{-1}\left(\sigma_{t}^{\prime}\right)^{-1} \phi_{t}$ where $\phi$ is the unique, progressively measurable process in the representation of the martingale

$$
M_{t} \equiv E\left(\int_{0}^{T} \xi_{s}\left(I\left(\left(y^{*} \Leftrightarrow \gamma_{s}^{*}\right) \xi_{s}\right) \Leftrightarrow e_{s}\right) d s \mid \mathcal{F}_{t}\right) \Leftrightarrow E\left(\int_{0}^{T} \xi_{s}\left(I\left(\left(y^{*} \Leftrightarrow \gamma_{s}^{*}\right) \xi_{s}\right) \Leftrightarrow e_{s}\right) d s\right) .
$$

On the event $\left\{X_{t}=0\right\}$ there is no investment in the stock: $\pi_{t}=0$.

At times when the constraint binds liquid wealth must be locally nondecreasing in order to keep meeting the constraint. This can only be achieved if investment in the stock market is null: on the event $\left\{X_{t}=0\right\}$ the optimal portfolio is $\pi_{t}=0$.

To shed further light on the nature of the solution to the constrained problem we next use the optimality conditions derived above to solve the example put forth in the introduction.

\subsection{An example with liquidity constraints.}

We now reconsider the example of Section 1.1, in the presence of a liquidity constraint. In order to solve the consumption-portfolio problem, we use the algorithm based on the backward procedure developed in section 3.4. Whenever the liquidity constraint binds the algorithm recomputes the multiplier at all successor nodes to ensure nonnegative liquid wealth. The next tree shows the evolution of the multiplier $(\gamma)$, optimal consumption $(c)$, end-of-period portfolio holdings $(\Delta, B)$, and beginning of period liquid wealth $(X)$. The Lagrange multiplier for the budget constraint in the presence of liquidity constraints is 1.0932 .

$$
\begin{aligned}
& \gamma^{u u}=0.9833 \\
& c^{u u}=2.5495 \\
& \gamma^{u}=0.6959 \\
& c^{u}=1.5033 \\
& X^{u}=0 \\
& \begin{aligned}
\Delta^{u} & =\Leftrightarrow .4004 \quad \nearrow \\
B^{u} & =.6006
\end{aligned} \\
& X^{u u}=0 \\
& \gamma^{u d}=0.6959 \\
& c^{u d}=1.3602 \\
& \gamma=0 \\
& \begin{array}{ll}
c=0.9707 & \Delta=\Leftrightarrow .21583 \quad \nearrow \\
X=0 & B=.25764 \quad \searrow
\end{array} \\
& \gamma^{d}=0 \\
& c^{d}=0.8783 \\
& X^{u}=0.0627 \\
& \Delta^{d}=\Leftrightarrow .7068 \\
& B^{d}=.7068 \\
& X^{u d}=0.2002 \\
& \gamma^{d u}=0.4526 \\
& c^{d u}=1.16 \\
& X^{d u}=0 \\
& \gamma^{d d}=0 \\
& c^{d d}=0.7947 \\
& X^{d d}=0.0728
\end{aligned}
$$


Consider nodes $(u),(u u)$ and compare them with their equivalents in the unconstrained tree in Section 1.1. At these nodes, the agent experiences good news regarding the realization of his labor income relative to the

previous period. The unconstrained portfolio value is then negative because the agent tailors his portfolio so as to transfer funds out of these nodes towards southern nodes of the tree with relatively less favorable income realizations.

However, the constrained portfolio value is zero at these nodes: the agent cannot transfer as much wealth from his high income realizations in nodes $(u, u u)$ to the rest of the tree as he would like, i.e. he cannot borrow against his future labor income. A consequence is that consumption in the states $(u, u u)$ has to increase relative to the unconstrained case, in which the individual can hedge without restrictions. We thus have $\left(c^{u}, c^{u u}\right)=(1.5033,2.5495)$ in the constrained case versus $\left(c^{u}, c^{u u}\right)=(1.3264,1.4659)$ in the unconstrained case of Section 1.1.

Note that this extra consumption "gulp" (relative to the unconstrained case) is due to the inability to hold an aggregate short position at $u$. This is a source of path dependency in optimal behavior (note that the tree above is not recombining while the tree in the unconstrained case is recombining). The occurrence of a binding constraint at any of these nodes will permanently decrease the marginal valuation of wealth at follower nodes, in the sense that the individual will attempt to consume this extra consumption "gulp" by smoothing it across follower nodes.

For example, consider node $(u d)$ in which unconstrained and constrained wealth are positive (i.e. $X^{u d}=0.0402$ and $X^{u d}=0.2002$, respectively). In the constrained case, the agent reaches that node after experiencing a binding constraint at the prior node $(u)$, and even though he faces exactly the same endowment and security returns that result in positive wealth in the unconstrained case, he ends up consuming more: compare $c^{u d}=1.3602$ versus $c^{u d}=1.2002$ in the unconstrained case. The increase in the Lagrange multiplier process $\gamma$ along the tree captures this random but permanent decreases in the marginal utility of income.

Of course, the inability to transfer wealth out of states $(u)$ and $(u u)$ by constructing a portfolio with a negative payoff in those states implies that the investor is unable to compensate for his low endowment in states $(d, d u, d d)$. Consumption at these nodes is therefore lower than in the unconstrained case. Furthermore, as noted above, path dependency arises in the consumption decision. For example, note that the portfolio value at $(d u)$ is zero. Since the investor is unable to transfer wealth out of $(u, u d)$, he tries to transfer wealth out of the next best node, which is $(d u)$, towards $(d, d d)$. To achieve this, the best he can do is to drive his wealth down to zero at the beginning of period three at node $(d u)$, and consume his full endowment $c^{d u}=e^{d u}=e^{u d}=1.16$ there. Thus, due to the path dependency, the constraint binds at $(d u)$ even though wealth was strictly positive there in the unconstrained case. Finally, at time zero he consumes an amount which leaves him with zero financial needs for future net consumption. This will be reflected in a larger multiplier value for the static budget constraint (3), namely 1.0932 versus 0.6545 . This implies that even though the constraint never binds, the consumption and portfolio behavior in the constrained and unconstrained case will be different (for example, compare the paths $\rightarrow d \rightarrow d d$ in the constrained and unconstrained cases).

In summary, liquidity constraints segment the uncertainty tree into subtrees. The nodes at which the constraint binds and their successors are treated in isolation of the rest of the tree for consumption smoothing purposes. Transfers of wealth then take place among the remaining nodes of the tree, which form an independent system. In the example above, the nodes $(u, u u, u d)$ form a closed subtree: wealth cannot be shifted from these good states toward bad states of nature. As a result, the wealth levels in the remaining nodes of the tree are lower in the constrained case than in the unconstrained case. This explains why the relative prices of consumption in different states will not be ex-ante equal to the marginal rate of substitution of consumption in these states. This provides a rationale to a potentially counterintuitive result: at times at which the liquid wealth of the consumer hits zero, he actually boosts his consumption level in a permanent fashion. Of 
course, this is compensated by smaller consumption levels in early age, almost everywhere relative to the unconstrained case. Note also that the constraint has a substantial impact on the individual: the ex-ante utility is $\Leftrightarrow 1.1722$ in the constrained case versus $\Leftrightarrow 0.9981$ in the unconstrained case. Thus, as can be verified, the individual would be willing to give up around $26 \%$ of his endowment at date 0 in order to avoid the constraint.

\subsection{The consumption function for CRRA.}

Under the assumption of power utility function the consumption function is

$$
c_{t}=\frac{X_{t}+E_{t}\left[\int_{t}^{T} \xi_{t, s} e_{s} d s\right]}{E_{t}\left[\int_{t}^{T} \xi_{t, s}^{1-1 / R}\left(\frac{y-\gamma_{s}}{y-\gamma_{t}}\right)^{-1 / R} d s\right]}
$$

The following properties hold.

Proposition 8 Suppose that the individual has constant relative risk aversion.

(i) Consider an economy in which unconstrained consumption is an increasing function of relative risk aversion. In this context constrained consumption may be humped (increasing-decreasing).

(ii) The constrained consumption function is homogeneous of degree one in initial endowment and wealth: $c\left(k X_{t}, k e_{t}, t\right)=k c\left(X_{t}, e_{t}, t\right)$.

Unconstrained consumption is increasing in relative risk aversion if the interest rate is sufficiently large relative to the market price of risk. For the model with constant coefficients a necessary and sufficient condition is $r>\left(\frac{1}{2} \Leftrightarrow 1 / R\right) \theta^{2}$. In the presence of a liquidity constraint consumption increases for low levels of risk aversion. Liquid wealth at future nodes is reduced and this eventually leads to a binding constraint. When the constraint binds at nodes that were previously unconstrained the subtrees issued from these nodes become segmented. Consumption smoothing then takes place over the remainder of the tree. The net result is that consumption eventually falls in order to allow reallocations over less favorable nodes of the remainder of the tree. Figure 1 illustrates this phenomenon.

The homogeneity property relative to total wealth follows from the homogeneity of the Lagrange multipliers with respect to multiplicative changes in endowments and liquid wealth. This result extends the standard property for the unconstrained policy.

\section{Equilibrium.}

In this section we show the existence of a competitive equilibrium (section 4.1), provide a characterization in terms of an "aggregator" (section 4.2), examine the equilibrium interest rate and the equity premium (section 4.3) and provide numerical estimates for power utility function (section 4.4).

\subsection{Existence of equilibrium.}

Consider the economy of section 2 with one unconstrained and one constrained agents (agents 1 and 2 , respectively). Let $C=D+e$ denote aggregate consumption. We suppose that aggregate endowment $e=e^{1}+e^{2}$ follows the Ito process

$$
d e_{t}=e_{t}\left[\mu_{t}^{e} d s+\sigma_{t}^{e} d W_{t}\right]
$$

where $\left(\mu^{e}, \sigma^{e}\right)$ are progressively measurable process. The aggregate consumption process $C=$ $D+e$ has drift $\mu^{c}$ and volatility coefficient $\sigma^{c}$ given by 
Figure 1: Parameter values: $r=.1, \theta=.3, \sigma=.2, \mu_{e}=.1, \sigma_{e}=.2, e_{0}=10, S_{0}=100$. Risk aversion between .02 and 3 .

$$
C \mu^{c}=D \mu^{D}+e \mu^{e} \quad \text { and } \quad C \sigma^{c}=D \sigma^{D}+e \sigma^{e} .
$$

To prove existence of equilibrium we need to solve the equations that describe market clearing and individual rationality, namely:

$$
\begin{gathered}
C_{t}=I_{1}\left(y^{1} \xi_{t}\right)+I_{2}\left(\left(y^{2} \Leftrightarrow \gamma_{t}\right) \xi_{t}\right) \\
\xi_{t} X_{t}^{2}=E\left(\int_{t}^{T} \xi_{s}\left[I_{2}\left(\left(y^{2} \Leftrightarrow \gamma_{s}\right) \xi_{s}\right) \Leftrightarrow e_{s}^{2}\right] d s \mid \mathcal{F}_{t}\right) \geq 0, \quad t \in[0, T] \\
X_{t}^{2} d \gamma_{t}=0, \gamma \text { nondecreasing null at } 0 \\
E \int_{0}^{T} \xi_{s}\left[I_{1}\left(y^{1} \xi_{s}\right) \Leftrightarrow e_{s}^{1}\right] d s=n^{1} S_{0}, \quad y^{1} \geq 0 \\
E \int_{0}^{T} \xi_{s}\left[I_{2}\left(\left(y^{2} \Leftrightarrow \gamma_{s}\right) \xi_{s}\right) \Leftrightarrow e_{s}^{2}\right] d s=n^{2} S_{0}, \quad y^{2} \geq 0 .
\end{gathered}
$$

By Walras law one of the budget constraints (32)-(33) is redundant. We can then set $y^{1}=1$, define $y \equiv y^{2}$ and solve the remaining system for $(y, \gamma, \xi)$. Solving the market clearing condition (29) yields the state price density (SPD)

$$
\xi_{t}=g\left(C_{t}, y \Leftrightarrow \gamma_{t}\right) \equiv g_{t}\left(y \Leftrightarrow \gamma_{t}\right)
$$


where $g(\cdot)$ is the inverse aggregate demand function with respect to the SPD, i.e. $g(\cdot)$ is the unique solution of $C_{t}=I_{1}\left(g\left(C_{t}, y \Leftrightarrow \gamma_{t}\right)\right)+I_{2}\left(\left(y \Leftrightarrow \gamma_{t}\right) g\left(C_{t}, y \Leftrightarrow \gamma_{t}\right)\right)$. Note that $g(\cdot)$ depends on the net shadow price of the constraints $x=y \Leftrightarrow \gamma$. Define the process $h_{t}(x)$ which represents the value of net consumption at date $t$ conditional on the net shadow price as

$$
h_{t}(x)=g_{t}(x)\left[I_{2}\left(x g_{t}(x)\right) \Leftrightarrow e_{t}^{2}\right] .
$$

Substituting (35) in (30),(31) and (33) leaves us with the system

$$
\begin{gathered}
\xi_{t} X_{t}^{2}=E\left(\int_{t}^{T} h_{s}\left(y \Leftrightarrow \gamma_{s}\right) d s \mid \mathcal{F}_{t}\right) \geq 0, \quad t \in[0, T] \\
X_{t}^{2} d \gamma_{t}=0, \gamma \text { nondecreasing null at } 0 \\
E \int_{0}^{T} h_{s}\left(y \Leftrightarrow \gamma_{s}\right) d s=n^{2} S_{0}, \quad y \geq 0 .
\end{gathered}
$$

The system (36)-(38) is similar in structure to the system of equations characterizing the optimal consumption policy in the static problem. As we show in the appendix, the same methodology can then be applied to prove the existence of a solution. This yields the existence of the competitive equilibrium and the following characterization.

Theorem 9 Consider the economy $\mathcal{E}$ of section 2. A competitive equilibrium $\left(\left(c^{i}, \pi^{i}\right)_{i \in C \cup U}\right.$, $(S, \mu, A, \sigma), r)$ exists.

Theorem 9 is proved as a particular case of the $N$-agent equilibrium in Appendix C. Note that our construction provides the equilibrium state price density in terms of the inverse aggregate demand function and the shadow prices of the budget and liquidity constraints. In the next section, we show that equilibrium prices in our economy are fully characterized by the SPD $\xi_{t}=g\left(C_{t}, y \Leftrightarrow \gamma_{t}\right)$ where $g(\cdot)$ is the inverse aggregate demand function defined above and the multipliers $(y, \gamma)$ uniquely solve (36)-(38). This might come as a surprise, since aggregation results are only known to hold in complete markets economies or economies with incomplete markets but restricted diversity among agents. We therefore elaborate on this point in the next section. In further sections, we will detail the structure of other equilibrium quantities and prices that can be inferred from this SPD.

\subsection{A representative agent characterization of equilibrium.}

In economies with complete markets and diverse agents the second welfare theorem establishes the existence of a no-trade equilibrium with identical pricing kernel. One can use this construction to prove existence of equilibrium (Neigishi (1960)) or to characterize asset prices without demand aggregation (Constantinides (1982)). Here we use the construction to characterize asset prices. In independent work, Cuoco and He (1994) also applied a similar representative agent methodology in an incomplete market model situation (though their source of incompleteness is different from ours).

A feasible allocation is a non-negative array of processes $\left(x^{i}\right)$ such that $\sum_{i} x_{t}^{i}=\sum_{i} c_{t}^{i}$. For simplicity here, take initial wealth to be zero. 
Proposition 10 Suppose that $\left(\left(c^{i}, \pi^{i}\right)_{i \in C \cup U},(S, \mu, A, \sigma), r\right)$ is an equilibrium for the economy $\mathcal{E}$. Then the competitive allocation $\left(c^{i}\right)$ solves the problem

$$
\begin{aligned}
U(C) & \equiv \sup _{\left\{x^{i}\right\}} E\left(\int_{0}^{T} \sum_{i \in C \cup U} \alpha_{t}^{i} u^{i}\left(x_{t}^{i}, t\right) d t\right) \quad \text { s.t. } \\
\sum_{i \in C \cup U} x_{t}^{i} & =\sum_{i \in C \cup U} c_{t}^{i} \equiv C_{t} \quad \text { a.s. }
\end{aligned}
$$

with $\alpha_{t}^{i} \equiv 1 /\left(y^{i} \Leftrightarrow \gamma_{t}^{i}\right)$ where $y^{i}, \gamma^{i}$ are the multipliers associated with the individual control problems, for $i \in C \cup U$ (we maintain the convention $\gamma_{t}^{i}=0$ for $i \in U$ ). Moreover, $(c, \pi)=(C, 1)$ is a no-trade equilibrium for a single agent economy where the agent has the preferences $U$ in (39).

One can prove that the maximum of (39) is achieved at

$$
c_{t}^{i}=I^{i}\left(\frac{H\left(C_{t}, t ; y, \gamma_{t}\right)}{\alpha_{t}^{i}}\right)
$$

where $H\left(x, t ; y, \gamma_{t}\right)$ is the inverse of the continuous and decreasing function $\sum_{i} I^{i}\left(x / \alpha_{t}^{i}\right)$ for $x>0$. Substituting back in (39) yields

$$
U^{\prime}\left(C_{t}\right)=H\left(C_{t}, t ; y, \gamma_{t}\right)
$$

i.e., the pricing kernel $H$, identified from (40), is the marginal utility of the representative agent $U^{\prime}$. In the next sections we will make extensive use of the fact that the market price of risk is driven by the risk aversion of the representative agent. Dybvig (1983) proved the same result for a complete markets economy:

Proposition 11 The relative risk aversion coefficient of the representative agent with utility function (39) is the weighted harmonic average of the relative risk aversion coefficients of the individuals in the economy where the weights are the respective consumption shares.

Note that the previous result implies that, in equilibrium, the price of risk (expected return per unit of volatility) is bounded above and below by the largest and smallest coefficient of relative risk aversion of the individuals in the economy, respectively. If we understand that the much discussed "equity premium puzzle" makes reference to an abnormally high expected stock return per unit of risk (the Sharpe ratio) rather than the level of the expected return ${ }^{5}$, the above aggregation result in Proposition 11 states that it is futile to attempt to explain the puzzle through liquidity constraints or incomplete markets in general: it is impossible to generate an aggregate risk aversion which is larger than the relative risk aversion of all individuals in the economy.

\subsection{Interest rate and equity premium with liquidity constraints.}

In order to describe the equilibrium interest rate and prices it is convenient to use the risk aversion coefficient $\left(R^{a}\right)$ and prudence coefficient $\left(P^{a}\right)$ of the representative agent constructed in the previous section. These are respectively given by

$$
\begin{aligned}
R_{t}^{a} & =C_{t}\left[\sum_{i} \frac{I_{i}\left(\left(y^{i} \Leftrightarrow \gamma_{t}^{i}\right) \rho_{t} \xi_{t}\right)}{R^{i}\left(\left(y^{i} \Leftrightarrow \gamma_{t}^{i}\right) \rho_{t} \xi_{t}\right)}\right]^{-1}=C_{t}\left[\sum_{i} \frac{c_{t}^{i}}{R_{t}^{i}}\right]^{-1} \\
P_{t}^{a} & =\frac{\left(R_{t}^{a}\right)^{2}}{C_{t}} \sum_{i} \frac{P^{i}\left(\left(y^{i} \Leftrightarrow \gamma_{t}^{i}\right) \rho_{t} \xi_{t}\right) I_{i}\left(\left(y^{i} \Leftrightarrow \gamma_{t}^{i}\right) \rho_{t} \xi_{t}\right)}{R^{i}\left(\left(y^{i} \Leftrightarrow \gamma_{t}^{i}\right) \rho_{t} \xi_{t}\right)^{2}}=\frac{\left(R_{t}^{a}\right)^{2}}{C_{t}} \sum_{i} \frac{P_{t}^{i} c_{t}^{i}}{\left(R_{t}^{i}\right)^{2}} .
\end{aligned}
$$

\footnotetext{
${ }^{5}$ The rationale for defining the equity premium puzzle through the Sharpe ratio lies in the fact that, keeping other quantities constant, the level of expected return of its stock is affected by the leverage assumed by the firm.
} 
where $R^{i}$ and $P^{i}$ represent the relative risk aversion and relative prudence coefficients of agent $i$. Our next result details the structure of the competitive equilibrium.

Theorem 12 Consider the economy $\mathcal{E}$ of section 2 with $u^{i}(c, t)=\exp (\Leftrightarrow \beta t) u^{i}(c)$ and suppose that Assumption 1 holds with $\theta_{t}=R_{t}^{a} \sigma_{t}^{c}$. The equilibrium interest rate and equity premium are

$$
\begin{aligned}
d R_{t} & =r_{t} d t+d A_{t} \\
r_{t} & =\beta+R_{t}^{a} \mu^{c} \Leftrightarrow \frac{1}{2} R_{t}^{a} P_{t}^{a}\left(\sigma^{c}\right)^{2} \\
d A_{t} & =\Leftrightarrow \frac{R_{t}^{a}}{R_{t}^{2}} \frac{c_{t}^{2}}{C_{t}} \frac{d \gamma_{t}}{y \Leftrightarrow \gamma_{t}} \\
\mu_{t} \Leftrightarrow r_{t} & =R_{t}^{a} \sigma_{t} \sigma_{t}^{c} .
\end{aligned}
$$

The stock price satisfies the present value formula

$$
S_{t}=E\left[\int_{t}^{T} \frac{g_{v}}{g_{t}} D_{v} d v \mid \mathcal{F}_{t}\right]
$$

where $g_{v}=g\left(D_{v}, y \Leftrightarrow \gamma_{v}\right)$ and its volatility is the unique process $\sigma$ such that for all $t \in[0, T]$

$$
E\left[\int_{0}^{T} g_{v} D_{v} d v \mid \mathcal{F}_{t}\right] \Leftrightarrow E\left[\int_{0}^{T} g_{v} D_{v} d v\right]=\int_{0}^{t} g_{v} S_{v}\left(\sigma_{v} \Leftrightarrow R_{v}^{a} \sigma_{v}^{c}\right) d W_{v} .
$$

In equilibrium the riskless cumulative rate has two components. The first is a locally riskless interest rate $r$ which has the familiar structure (43). As usual $r$ is positively related to the expected consumption growth rate and negatively related to aggregate consumption risk if the aggregator exhibits positive prudence. Unlike models with portfolio constraints or incomplete markets, the cumulative interest rate also has a singular component $A$, described in (44). This component is tied to the occurrence of a binding liquidity constraint. Whenever the constraint binds the consumption demand (savings demand) of the agent experiences a permanent predictable increase (decrease). The cumulative interest rate naturally reflects the increased demand: its fall is just enough to provide an adequate incentive to reduce consumption immediately. The extent of the decrease in cumulative interest rate is related to the size of the net multiplier $y \Leftrightarrow \gamma$. If the net multiplier is small, past consumption deferral has been substantial enough to ensure that the liquidity constraint is satisfied. If the liquidity constraint binds, the marginal value of wealth decreases and the additional consumption increase required to match this decrease becomes large (since marginal utility is very low). The required decrease in the cumulative riskfree return becomes comparatively large. ${ }^{6}$ The analysis above, thus, shows that liquidity constraints tend to reduce the cumulative riskless return relative to an unconstrained economy. The incorporation of these constraints may then help to resolve the riskfree rate puzzle (Weil (1990)).

Since the interest rate reflects all the deterministic components in the growth rate of the marginal utility of the aggregator it is not surprising to realize that the risk premium of the stock (45) does not depend directly on the liquidity constraint. Indeed this risk premium has the usual structure: it satisfies the consumption CAPM. Indirect effects of the liquidity constraint, however, affect the intertemporal properties of the equity premium because the risk aversion parameter of the aggregator is a composite of the risk aversions of all agents in the economy and therefore reflects the distribution of consumption across agents. Whenever the constraint binds the aggregator's weight reallocates consumption across agents and this generates singular components in the evolution of the equity premium. Does this mean that liquidity constraints

\footnotetext{
${ }^{6}$ Singular components in cumulative interest rates also arise when marginal utility of agents is bounded at zero (see Karatzas et al (1991)).
} 
can be invoked to justify the empirical magnitude of the Sharpe ratio? The answer is negative. Indeed, the Sharpe ratio has an empirical magnitude of about .37 (using Mehra and Prescott's (1985) numbers) Since consumption growth's volatility is .0357 this implies an aggregator's risk aversion of 10.4 (see (45)). However, this number is unrealistic since the aggregator's risk aversion is bounded above by the highest risk aversion of the agents populating the economy.

\subsection{Examples}

In this section we provide examples that can be solved in closed form and which illustrate the effects of liquidity constraints described above. In the first example, agents have homogeneous constant relative risk aversion parameter: the cumulative interest decreases while the Sharpe ratio is unaffected. In the second example, relative risk aversions depend on the consumption levels and distributional effects affect equilibrium prices.

\subsubsection{Example 1.}

Consider a simple economy with 2 agents with homogeneous and constant relative risk aversion $R$. The aggregator's risk aversion and prudence coefficients are $R_{t}^{a}=R$ and $P_{t}^{a}=1+R$. The equilibrium Sharpe ratio which equals

$$
\frac{\mu_{t} \Leftrightarrow r_{t}}{\sigma_{t}}=R \sigma_{t}^{c}
$$

is unaffected by the liquidity constraint. The cumulative interest is given by

$$
\begin{aligned}
d R_{t} & =r_{t} d t+d A_{t} \\
r_{t} & =\beta+R \mu_{t}^{c} \Leftrightarrow \frac{1}{2} R(1+R)\left(\sigma_{t}^{c}\right)^{2} \\
d A_{t} & =\Leftrightarrow \frac{c_{t}^{2}}{C_{t}} \frac{d \gamma_{t}}{y \Leftrightarrow \gamma_{t}} .
\end{aligned}
$$

Hence, the interest rate $r$ is the same while the cumulative interest $R$ decreases relative to an economy without the liquidity constraint but identical in all other respects.

\subsubsection{Example 2.}

Consider now an economy with 2 agents with relative risk aversion coefficients $R_{t}^{1}=R^{1}\left(c_{t}^{1}\right)$ and $R_{t}^{2}=R^{2}\left(c_{t}^{2}\right)$. The aggregator's risk aversion coefficient is

$$
R_{t}^{a}=C_{t}\left[\frac{c_{t}^{1}}{R_{t}^{1}}+\frac{c_{t}^{2}}{R_{t}^{2}}\right]^{-1}
$$

Using $c_{t}^{1}+c_{t}^{2}=C_{t}$ we get

$$
\frac{\partial R_{t}^{a}}{\partial c_{t}^{2}}<0 \text { iff } \frac{R_{t}^{1} \Leftrightarrow R_{t}^{2}}{R_{t}^{1} R_{t}^{2}} \Leftrightarrow\left[\frac{c_{t}^{2}}{\left(R_{t}^{2}\right)^{2}} \frac{\partial R_{t}^{2}}{\partial c_{t}^{2}} \Leftrightarrow \frac{c_{t}^{1}}{\left(R_{t}^{1}\right)^{2}} \frac{\partial R_{t}^{1}}{\partial c_{t}^{1}}\right]>0 .
$$

Under this condition the aggregator's risk aversion increases in the early stages of the liquidity constrained economy relative to an unconstrained economy. The equilibrium Sharpe ratio will be larger as well. Sufficient conditions for (48) to hold are decreasing relative risk aversion of constrained agents, combined with increasing relative risk aversion of unconstrained agents and $R^{1} \geq R^{2}$ at the unconstrained allocation. 


\section{Liquidity constraints and incomplete markets.}

In this section we outline an extension of our results to incomplete markets. In this environment liquidity constraints combine with the failure of full insurance to determine equilibrium prices and the consumption allocation.

We suppose that the uncertainty is represented by the 2-dimensional Wiener process $\bar{W}=$ $\left(\bar{W}_{1}, \bar{W}_{2}\right)$ defined on $(\Omega, \mathcal{F})$. The filtration $\mathcal{F}_{(\cdot)}$ is the natural filtration generated by $\bar{W}$. The dividend process of the stock and the aggregate endowment processes are

$$
\begin{aligned}
d D_{t} & =D_{t}\left[\mu_{t}^{D} d t+\sigma_{t}^{D} d \bar{W}_{1 t}\right], \quad D_{0} \text { given. } \\
d e_{t} & =e_{t}\left[\mu_{t}^{e} d t+\sigma_{1 t}^{e} d \bar{W}_{1 t}+\sigma_{2 t}^{e} d \bar{W}_{2 t}\right], \quad e_{0} \text { given }
\end{aligned}
$$

where $\left(\mu^{D}, \mu_{t}^{e}, \sigma^{D}, \sigma_{1}^{e}, \sigma_{2}^{e}\right)$ are progressively measurable processes, i.e. they may depend on both Brownian motions $\bar{W}_{1}$ and $\bar{W}_{2}$. Aggregate consumption $C=D+e$ evolves according to

$$
d C_{t}=C_{t}\left[\mu_{t}^{c} d t+\sigma_{1 t}^{c} d \bar{W}_{1 t}+\sigma_{2 t}^{c} d \bar{W}_{2 t}\right], \quad C_{0} \text { given. }
$$

Let $\sigma_{t}^{c}=\left(\left(\sigma_{1 t}^{c}\right)^{2}+\left(\sigma_{2 t}^{c}\right)^{2}\right)^{\frac{1}{2}}$ and define the new processes ${ }^{7}$

$$
\left\{\begin{array}{rl}
d W_{1 t} & =\frac{1}{\sigma_{t}^{c}}\left[\sigma_{1 t}^{c} d \bar{W}_{1 t}+\sigma_{2 t}^{c} d \bar{W}_{2 t}\right] \\
d W_{2 t} & =\frac{1}{\sigma_{t}^{c}}\left[\sigma_{2 t}^{c} d \bar{W}_{1 t} \Leftrightarrow \sigma_{1 t}^{c} d \bar{W}_{2 t}\right] .
\end{array} .\right.
$$

In this new system of coordinates aggregate consumption and aggregate endowment become

$$
\begin{aligned}
d C_{t} & =C_{t}\left[\mu_{t}^{c} d t+\sigma_{t}^{c} d W_{1 t}\right] \\
d e_{t} & =e_{t}\left[\mu_{t}^{e} d t+\frac{1}{\sigma_{t}^{c}}\left[\left(\sigma_{1 t}^{e} \sigma_{1 t}^{c}+\sigma_{2 t}^{e} \sigma_{2 t}^{c}\right) d W_{1 t}+\left(\sigma_{1 t}^{e} \sigma_{2 t}^{c} \Leftrightarrow \sigma_{2 t}^{e} \sigma_{1 t}^{c}\right) d W_{2 t}\right]\right.
\end{aligned}
$$

The stock price satisfies

$$
d S_{t}+D_{t} d t=S_{t}\left[d m_{t}+\sigma_{1 t} d W_{1 t}+\sigma_{2 t} d W_{2 t}\right]
$$

subject to some initial value $S_{0}$, where $m$ and $\sigma$ are progressively measurable and $d m_{t}=\mu_{t} d t+d A_{t}$. The riskless return is $d R_{t}=r_{t} d t+d A_{t}$. The remaining structure of the economy as well as the definitions of individual rationality and equilibrium are the same as in section 2 .

In this economy there are 2 sources of uncertainty but only one risky stock. Note that (53) allows for the possibility that the individual idiosyncratic income is not spanned by traded assets. Thus the market is incomplete even in the absence of any liquidity constraints. The initial value of the stock $S_{0}$, the coefficients $\left(m, \mu, A, \sigma_{1}, \sigma_{2}\right)$, and the values of $R$ and $r$ are endogenous. Note, in particular, that individual rationality combined with market clearing will determine the

\footnotetext{
${ }^{7}$ This definition implies $\mathcal{F}_{(\cdot)}^{W} \subseteq \mathcal{F}_{(\cdot)}^{\bar{W}}$ (the filtration generated by $W$ is contained in the one generated by $\bar{W}$ ). It is also straightforward to verify that $W_{1}$ and $W_{2}$ are independent Brownian motion processes. Furthermore provided $\sigma_{t}^{c}>0$ for all $t \in[0, T]$ we can invert this relation and write the following stochastic differential equations for $\bar{W}$,

$$
\left\{\begin{array}{l}
d \bar{W}_{1 t}=\frac{1}{\sigma_{t}^{c}}\left[\sigma_{1 t}^{c} d W_{1 t}+\sigma_{2 t}^{c} d W_{2 t}\right] \\
d \bar{W}_{2 t}=\frac{1}{\sigma_{t}^{c}}\left[\sigma_{2 t}^{c} d W_{1 t}-\sigma_{1 t}^{c} d W_{2 t}\right]
\end{array} .\right.
$$

If the coefficients $\left(\frac{\sigma_{1 t}^{c}}{\sigma_{t}^{c}}, \frac{\sigma_{2 t}^{c}}{\sigma_{t}^{c}}\right)$ satisfy standard Lipschitz and Growth conditions this SDE has a unique strong solution. This implies $\mathcal{F}_{(\cdot)}^{\bar{W}} \subseteq \mathcal{F}_{(\cdot)}^{W}$. Combining these two inclusions gives $\mathcal{F}_{(\cdot)}^{W}=\mathcal{F}_{(\cdot)}^{\bar{W}}$. Hence there is no loss of generality if we take $W$ as our primitive.
} 
volatility structure $\left(\sigma_{1}, \sigma_{2}\right)$ of the stock price: the class of price processes $(54)$ is sufficiently large to accommodate an instantaneous impact of both Brownian motion processes on the stock price.

Since markets are incomplete agents have individual valuations of the risks affecting the economy. Assuming that $\sigma_{1} \neq 0$ we can define the individual prices of risk from $i$ 's point of view

$$
\theta^{i}=\left[\begin{array}{l}
\theta_{1}^{i} \\
\theta_{2}^{i}
\end{array}\right]=\left[\begin{array}{ll}
\sigma_{1} & \sigma_{2} \\
0 & 1
\end{array}\right]^{-1}\left[\begin{array}{l}
\mu \Leftrightarrow r \\
\alpha^{i} \Leftrightarrow r
\end{array}\right]
$$

where $\alpha^{i}$ represents the shadow price of the incomplete market constraint, i.e. it represents the drift of a fictitious individualized security with return $d f_{t}=f_{t}\left[\alpha_{t}^{i} d t+d W_{2 t}\right]$. The incomplete market restriction can be viewed as a portfolio constraint which precludes any investment in this fictitious security. Hence if $\left(\pi^{i}, \pi_{f}^{i}\right)$ denotes the demand for investment in the stock and the fictitious asset respectively, the incomplete markets constraint can be summarized by the condition $\pi_{f}^{i}=0$. In this setting the counterpart of Assumption 1 is

Assumption 1': The individual prices of risk $\theta^{i}$ satisfy $E \exp \left(\frac{1}{2} \int_{0}^{T}\left\|\theta_{t}^{i}\right\|^{2} d t\right)<\infty, i=1,2$.

The dynamic problem of a liquidity constrained individual is

$$
\sup _{(c, \pi)} U(c) \quad \text { s.t. } \quad(c, \pi) \in \mathcal{A}
$$

The corresponding static problem becomes

$$
\begin{gathered}
\sup _{c} U(c) \quad \text { s.t } \\
\left\{\begin{array}{l}
c \geq 0, \text { progressively measurable and integrable } \\
\xi_{t}^{i} X_{t}=E\left(\int_{t}^{T} \xi_{s}^{i}\left(c_{s} \Leftrightarrow e_{s}\right) d s \mid \mathcal{F}_{t}\right) \geq 0 \quad t \in[0, T] \\
E\left(\int_{0}^{T} \xi_{t}^{i}\left(c_{t} \Leftrightarrow e_{t}\right) d t\right)=n S_{0}, \\
\alpha \text { such that } X \in S p[S]
\end{array}\right.
\end{gathered}
$$

where $\xi^{i}$ is the state price density based on the market price of risk $\theta^{i}$ and where $S p[S]$ denotes the span generated by the process $S$, i.e. the span of $\int_{0}^{t}\left(\sigma_{1 t} d W_{1 t}+\sigma_{2 t} d W_{2 t}\right)$. The following proposition establishes the relationship between problems (55) and (56)-(57).

Proposition 13 Assume that assumption 1' holds and that u satisfies the conditions of assumption 2. If $(c, \pi)$ solves the dynamic problem (55), then there exists $\alpha$ such that $c$ solves the static problem (56)-(57). Conversely, if $(c, \alpha)$ solves the static problem (56)-(57), then there exists a portfolio policy $\pi$ such that $(c, \pi)$ solves the dynamic problem (55).

The static problem gives rise to the following Kuhn-Tucker first order conditions

$$
\left\{\begin{array}{l}
u_{c}\left(c_{t}, t\right)=\left(y \Leftrightarrow \gamma_{t}\right) \xi_{t}^{i} \\
E\left(\int_{0}^{T} \xi_{t}^{i}\left(c_{t} \Leftrightarrow e_{t}\right) d t\right)=n S_{0} \\
E\left(\int_{t}^{T} \xi_{s}^{i}\left(c_{s} \Leftrightarrow e_{s}\right) d s \mid \mathcal{F}_{t}\right) \geq 0 \\
E\left(\int_{0}^{T} X_{t} d \gamma_{t}\right)=0 \\
c_{t} \geq 0, y \geq 0 \\
\gamma \geq 0, \text { nondecreasing, null at } 0 \\
\alpha \text { such that } X \in S p[S] .
\end{array}\right.
$$


The only difference with the earlier formulation is the addition of the incompleteness constraint (last equation) to the set of relevant conditions.

Our next verification theorem shows that the conditions (58) are sufficient for the static optimization problem.

Theorem 14 Assume that assumptions 1' and 2 hold. Suppose that there exist progressively measurable processes $(c, \gamma, \alpha)$ and a constant $y$ such that $(c, \gamma, \alpha, y)$ solves (58). Then $c$ solves (56) subject to (57).

In order to characterize the competitive equilibrium we need to solve the equations that describe market clearing and individual rationality, namely:

$$
\begin{gathered}
C_{t}=I_{1}\left(y^{1} \xi_{t}^{1}\right)+I_{2}\left(\left(y^{2} \Leftrightarrow \gamma_{t}\right) \xi_{t}^{2}\right) \\
\xi_{t} X_{t}^{2}=E\left(\int_{t}^{T} \xi_{s}^{2}\left[I_{2}\left(\left(y^{2} \Leftrightarrow \gamma_{s}\right) \xi_{s}^{2}\right) \Leftrightarrow e_{s}^{2}\right] d s \mid \mathcal{F}_{t}\right) \geq 0, \quad t \in[0, T] \\
X_{t}^{2} d \gamma_{t}=0, \quad \gamma \text { nondecreasing null at } 0 \\
E \int_{0}^{T} \xi_{s}^{1}\left[I_{1}\left(y^{1} \xi_{s}^{1}\right) \Leftrightarrow e_{s}^{1}\right] d s=n^{1} S_{0}, \quad y^{1}>0 \\
E \int_{0}^{T} \xi_{s}^{2}\left[I_{2}\left(\left(y^{2} \Leftrightarrow \gamma_{s}\right) \xi_{s}^{2}\right) \Leftrightarrow e_{s}^{2}\right] d s=n^{2} S_{0}, \quad y^{2}>0 . \\
\alpha^{i} \text { s.t. } X^{i} \in S p[S]
\end{gathered}
$$

By Walras law one of the budget constraints (62)-(63) is redundant. We can then set $y^{1}=1$, define $y \equiv y^{2}$ and $\eta=\xi^{2} / \xi^{1}$ and solve the remaining system for $\left(y, \gamma, \xi^{1}, \eta\right)$. Solving the market clearing condition (59) yields the SPD

$$
\xi_{t}^{1}=g\left(C_{t}, y \Leftrightarrow \gamma_{t}, \eta_{t}\right) \equiv g_{t}\left(y \Leftrightarrow \gamma_{t}, \eta_{t}\right)
$$

where $g(\cdot)$ is the unique solution of $C_{t}=I_{1}\left(g\left(C_{t}, y \Leftrightarrow \gamma_{t}, \eta_{t}\right)\right)+I_{2}\left(\left(y \Leftrightarrow \gamma_{t}\right) g\left(C_{t}, y \Leftrightarrow \gamma_{t}, \eta_{t}\right) \eta_{t}\right)$. Define the process $h_{t}\left(x, \eta_{t}\right)$ which represents the value of net consumption at date $t$ conditional on the net shadow price of the constraint $x=y \Leftrightarrow \gamma_{t}$ as

$$
h_{t}\left(x, \eta_{t}\right)=g_{t}\left(x, \eta_{t}\right)\left[I_{2}\left(x g_{t}\left(x, \eta_{t}\right) \eta_{t}\right) \Leftrightarrow e_{t}^{2}\right] .
$$

Substituting (65) in (60),(61) and (63) leaves us with the system

$$
\begin{gathered}
\xi_{t} X_{t}^{2}=E\left(\int_{t}^{T} h_{s}\left(y \Leftrightarrow \gamma_{s}, \eta_{s}\right) d s \mid \mathcal{F}_{t}\right) \geq 0, \quad t \in[0, T] \\
X_{t}^{2} d \gamma_{t}=0, \quad \gamma \text { nondecreasing null at } 0 \\
E \int_{0}^{T} h_{s}\left(y \Leftrightarrow \gamma_{s}, \eta_{s}\right) d s=n^{2} S_{0}, \quad y \geq 0 . \\
\alpha^{i} \text { s.t. } X^{i} \in S p[S] \quad \text { for } i=1,2 .
\end{gathered}
$$

This system of equations is in part similar to the system of equations characterizing the complete markets' equilibrium. The impact of incompleteness is embedded in the last two constraints which require that the liquid wealth processes of agents lie in the asset span. For the multiagent economy with incomplete market and liquidity constraint equilibrium has the following structure. 
Theorem 15 Consider the multiagent economy of section 2 with general utility function $u_{i}(c), i=$ 1.2 satisfying assumption 2. Assume that an equilibrium exists such that assumption 1' holds for $i=1,2$. The equilibrium interest rate and asset risk premia satisfy

$$
\begin{gathered}
r_{t}=R_{t}^{a} \mu^{c} \Leftrightarrow \frac{1}{2} R_{t}^{a} P_{t}^{a}\left(\sigma_{t}^{c}\right)^{2} \Leftrightarrow \frac{1}{2} R_{t}^{a} \frac{\sigma_{1 t}^{2}+\sigma_{2 t}^{2}}{\sigma_{1 t}^{2}} \sum_{i}\left[\frac{c_{t}^{i} P_{t}^{i}}{C_{t}\left(R_{t}^{i}\right)^{2}}\left(\theta_{2 t}^{i}\right)^{2}\right]+R_{t}^{a} \frac{\sigma_{2 t} \sigma_{t}^{c}}{\sigma_{1 t}} \sum_{i}\left[\frac{c_{t}^{i} P_{t}^{i}}{C_{t}\left(R_{t}^{i}\right)^{2}} \theta_{2 t}^{i}\right] \\
d A_{t}=\Leftrightarrow \frac{R_{t}^{a}}{R_{t}^{2}} \frac{c_{t}^{2}}{C_{t}} \frac{1}{y \Leftrightarrow \gamma_{t}} d \gamma_{t} \\
\mu_{t} \Leftrightarrow r_{t}=R_{t}^{a} \sigma_{t}^{c} \sigma_{1 t}
\end{gathered}
$$

where

$$
R_{t}^{a}=\left[\sum_{i} \frac{c_{t}^{i}}{C_{t}} \frac{1}{R_{t}^{i}}\right]^{-1} \quad \text { and } \quad P_{t}^{a}=\left(R_{t}^{a}\right)^{2} \sum_{i} \frac{c_{t}^{i} P_{t}^{i}}{C_{t}\left(R_{t}^{i}\right)^{2}}
$$

The structure of equilibrium reflects the different frictions in the economy. The interest rate is directly affected by the liquidity constraint as in the earlier model with complete market. In addition incompleteness plays a role: the level of the interest rate and the response to shifts in aggregate consumption growth rate and volatility depend on the individual prices of $W_{2}$-risk.

The risk premium of the stock displays structural properties which are similar to the case of complete markets since it satisfies the consumption CAPM: in view of (71), only covariance with aggregate consumption commands a premium. The liquidity constraint again has an indirect effect on the size of the premium through the risk aversion of the aggregator and the distribution of consumption in the economy. Note that incompleteness has no effect on the structure of the consumption CAPM since the distribution of marginal utilities across agents, which represents the second factor in this economy, is orthogonal to the stock's returns.

Note also that incompleteness cannot rationalize the empirical size of the Sharpe ratio either: the aggregator's risk aversion is the same harmonic average of the agents risk aversions as in the case of liquidity constraints alone. Since $W_{1}$ and $W_{2}$ risks are uncorrelated, it is easy to see from (71) that the Sharpe ratio of the market is bounded above by $R_{t}^{a} \sigma_{t}^{c}$, which is the Sharpe ratio corresponding to the same economy but with complete markets. This indicates that an aggregate risk aversion of 10.4 can not be plausibly supported in this model.

Our last result specializes the model to homogeneous constant relative risk aversion.

Corollary 16 Consider the multiagent economy of this section with homogeneous constant relative risk aversion $u_{i}(c)=\frac{1}{1-R} c^{1-R}, i=1,2$ for $i=1,2$. Assume that an equilibrium exists such that Assumption 1' holds. The equilibrium interest rate and the equity premium satisfy

$$
\begin{aligned}
& r_{t}=R \mu^{c} \Leftrightarrow \frac{1}{2} R(1+R)\left(\sigma_{t}^{c}\right)^{2} \Leftrightarrow \frac{1}{2} \frac{1+R}{R} \frac{\sigma_{1 t}^{2}+\sigma_{2 t}^{2}}{\sigma_{1 t}^{2}} \sum_{i}\left[\frac{c_{t}^{i}}{C_{t}}\left(\theta_{2 t}^{i}\right)^{2}\right] \\
& d A_{t}=\Leftrightarrow \frac{c_{t}^{2}}{C_{t}} \frac{d \gamma_{t}}{y \Leftrightarrow \gamma_{t}} \\
& \mu_{t} \Leftrightarrow r_{t}=R \sigma_{t}^{c} \sigma_{1 t} .
\end{aligned}
$$

Both the incomplete market restriction and the liquidity constraint reduce the interest rate relative to the complete market case.

Two additional features of the competitive equilibrium are noteworthy in the case under consideration. First note that both the incomplete market and the liquidity constraint tend to reduce the interest rate relative to the frictionless case. Incompleteness has a negative impact since marginal utilities and the distribution of consumption across agents become unambiguously 
more volatile when relative risk aversion is constant and homogeneous. This reinforces the case for an explanation of the riskfree puzzle based on incomplete markets and liquidity constraints.

The second feature of equilibrium is the fact that the Sharpe ratio does not increase because of market incompleteness or liquidity constraints. Since the CCAPM holds and due to the orthogonality between $d W_{1}$ and $d W_{2}$ risks in (54), it is easy to see from the third equation in Corollary 16 that when markets are incomplete, the Sharpe ratio is bounded above by the Sharpe ratio that would otherwise hold if markets were complete and the agent faced no liquidity constraint.

In summary, our results indicate there is no hope for explanations of the equity premium puzzle based on incomplete markets or liquidity constraints as defined in this paper. This poses a problem for the literature that seeks to resolve this puzzle on the basis of ad-hoc portfolio constraints. Our point is that once these portfolio constraints are endogenized and made effectively wealth-dependent, the asset-pricing implications regarding risk premia change radically.

\section{Conclusions.}

In this paper we presented a general equilibrium analysis of an exchange economy in which some agents cannot market their income. We have shown that a liquidity constraint restricts the consumption allocation of an individual over the lifecycle. Constrained individuals will optimally defer consumption to the future in order to insure against liquidity shortages. At times when the constraint binds their consumption experiences permanent increases. The individual consumption behavior affects the structure of prices and the interest rate at equilibrium. Our analysis demonstrates that short term rates have a tendency to decrease in the presence of a liquidity constraint. Liquidity constraints may then help to resolve the riskfree rate puzzle. However, the valuation of risk does not seem to change much since the equilibrium price of risk is a positive and convex combination of the relative risk aversion coefficients of the agents in the economy. In any case neither market incompleteness nor liquidity constraints can be invoked to explain the empirical magnitude of the Sharpe ratio. In further work we will seek to investigate analytically and numerically the impact of liquidity constraints on the volatility of asset prices and on the average level of the equity premium.

The paper also includes several methodological contributions. The first of these is a new approach to the consumption problem in the presence of a liquidity constraint which leads to an explicit solution for the consumption policy and which lends itself to numerical implementation. The second contribution is a constructive approach to the determination of equilibrium in economies with liquidity constraints. The third methodological contribution is the design of a computational algorithm which enables us to handle the type of path-dependent problems that arise in the presence of liquidity constraints. The fourth methodological contribution consists of a local aggregation method, essentially different from Rubinstein (1974), that enables us to characterize the pricing kernel as the marginal utility of a representative agent in an incomplete markets economy. We foresee several other areas of applications of these methods, including macroeconomic and international asset pricing models involving barriers and constraints, and models incorporating default risk.

\section{References}

[1] Alvarez, F. and U. Jermann, "Asset Pricing when Risk Sharing is Limited by Default: A Theoretical Analysis" unpublished manuscript, University of Chicago, 1997.

[2] Basak, S. and D. Cuoco, "An Equilibrium Model with Restricted Stock Market Participation," Review of Financial Studies, 1997: 
[3] Breeden, D., "An Intertemporal Asset Pricing Model with Stochastic Consumption and Investment Opportunities," Journal of Financial Economics, 7, 1979: 265-296.

[4] Constantinides, G., J. Donaldson and R. Mehra, "Junior Can’t Borrow: a New Perspective on the Equity Premium Puzzle," Working Paper, University of Chicago, 1997.

[5] Constantinides, G. and D. Duffie, "Asset Pricing with Heterogeneous Consumers," Journal of Political Economy, 104, 1996: 219-240.

[6] Constantinides, G. "Intertemporal Asset Pricing with Heterogeneous Consumers and without Demand Aggregation" Journal of Business, 55, 1982: 253-267

[7] Cox, J. C. and C.-F. Huang, "Optimal Consumption and Portfolio Policies when Asset Prices follow a Diffusion Process," Journal of Economic Theory, 49, 1989: 33-83.

[8] Cox, J. C. and C.-F. Huang , "A Variational Problem Arising in Financial Economics," Journal of Mathematical Economics, 1991: 465-487.

[9] Cuoco, D. and H. He, "Dynamic Equilibrium in Infinite-Dimensional Economies with Incomplete Financial Markets," Working Paper, University of Pennsylvania, 1994.

[10] Cvitanic, J. and I. Karatzas, "Convex Duality in Constrained Portfolio Optimization," Annals of Applied Probability, 2, 1992: 767-818.

[11] Deaton, A. "Saving and Liquidity Constraints" Econometrica 59, 1991: 1221-1248

[12] Duffie, D., "Stochastic Equilibrium: Existence, Spanning Number and the 'No Gains From Trade' Hypothesis" Econometrica 54, 1986: 1161-84.

[13] Duffie, D. and C.-F. Huang, "Implementing Arrow-Debreu Equilibria by Continuous Trading of a Few Long Lived Securities" Econometrica 53, 1985: 1337-56.

[14] Duffie, D. and W. Zame, "The Consumption-Based Capital Asset Pricing Model," Econometrica, 57, 1989: 1279-1297.

[15] Dugundji, J. and Granas, A., Fixed Point Theory PWN Polish Scientific Publishers, Warszawa, 1982.

[16] Dunford, N. and J. Schwartz, Linear Operators. Part I: General Theory Wiley Interscience, New York, 1958.

[17] Dybvig, P. (1983): “An Explicit Bound on Individual Assets' Deviations from APT Pricing in a Finite Economy" Journal of Financial Economics 12, p.483-96

[18] El Karoui, N. and M. Jeanblanc-Picque "Optimization of Consumption with Labor Income," Working Paper, Université d'Evry, 1995.

[19] He, H. and H. Pages, "Labor Income, Borrowing Constraints, and the Equilibrium Asset Prices: A Duality Approach," Economic Theory, 3, 1995: 663-696.

[20] He, H. and N. Pearson, "Consumption and Portfolio Policies with Incomplete Markets and Short-Sale Constraints: The Infinite Dimensional Case," Journal of Economic Theory, 54, 1991: 259-304.

[21] Hindy, A., "Asset Prices under Solvency Constraints," Journal of Mathematical Economics, 24, 1995: 105-135.

[22] Holmes, Geometric Functional Analysis Springer Verlag, New York, 1975. 
[23] Karatzas, I,. Lakner, P., Lehoczky, J. and S. E. Shreve, "Equilibrium in a Simplified Dynamic, Stochastic Economy with Heterogeneous Agents" mimeo, Dep. of Mathematics, CarnegieMellon University, 1990.

[24] Karatzas, I., Lehoczky, J. P. and S. E. Shreve, "Optimal Portfolio and Consumption Decisions for a "Small Investor" on a Finite Horizon," SIAM Journal of Control and Optimization, 25, 1987: $1557-1586$.

[25] Karatzas, I., Lehoczky, J. P. and S. E. Shreve, "Existence and Uniqueness of Multi-Agent Equilibrium in a Stochastic, Dynamic Consumption/Investment Model," Mathematics of Operations Research, 15, 1990: 80- 128.

[26] Karatzas, I., Lehoczky, J. and Shreve, S., "Equilibrium Models with Singular Asset Prices" Mathematical Finance 3, 1991: 11-29.

[27] Karatzas, I., Lehoczky, J. P., Shreve S. E. and G-L. Xu, "Martingale and Duality Methods for Utility Maximization in an Incomplete Market," SIAM Journal of Control and Optimization, 29, 1991: 702-730.

[28] Karatzas, I., and S. E. Shreve, Brownian Motion and Stochastic Calculus, Springer-Verlag, New York, 1988.

[29] Kehoe, T. and D. Levine, "Debt-Constrained Asset Markets," Review of Economic Studies, 60, 1993: 868-888.

[30] Lucas, R., "Asset Prices in a Pure Exchange Economy," Econometrica, 46, 1978: 1429-1445.

[31] Luenberger, D., Optimization by Vector Space Methods Wiley, New York, 1969.

[32] Mehra, R. and E. C. Prescott, "The Equity Premium: A Puzzle," Journal of Monetary Economics, 15, 1985: 145-161.

[33] Merton, R. C., "Optimum Consumption and Portfolio Rules in a Continuous Time Model," Journal of Economic Theory, 3, 1971: 373-413.

[34] Merton, R. C., “An Intertemporal Capital Asset Pricing Model," Econometrica, 41, 1973: 867-888.

[35] Scheinkman, J. and L. Weiss, "Borrowing Constraints and Aggregate Economic Activity," Econometrica, 54, 1986: 23-45.

[36] Yosida, K. and E. Hewitt, "Finitely Additive Measures" Transactions of the American Mathematical Society 72, 1952:. 46-66.

[37] Weil, P., "Equilibrium Asset Prices with Undiversifiable Labor Income Risk," Journal of Economic Dynamics and Control, 1992: 769-790:

\section{Appendices.}

\subsection{Appendix A: proofs.}

Proof of Lemma 1: Using Itô's lemma, we can express (10) as

$$
\xi_{t} X_{t}^{i}=X_{0}^{i}+\int_{0}^{t} \xi_{s}\left(e_{s}^{i} \Leftrightarrow c_{s}^{i}\right) d s+\int_{0}^{t} \xi_{s}\left(\pi_{s}^{i} \sigma_{s} \Leftrightarrow X_{s}^{i} \theta_{s}\right) d W_{s}
$$


which implies that the processes

$$
Y_{t}^{i} \equiv \xi_{t} X_{t}^{i}+\int_{0}^{t} \xi_{s}\left(c_{s}^{i} \Leftrightarrow e_{s}^{i}\right) d s \quad \text { and } \quad Z_{t}^{i} \equiv Y_{t}^{i}+E\left(\int_{0}^{T} \xi_{s} e_{s}^{i} d s \mid \mathcal{F}_{t}\right)
$$

are continuous local martingales. Now, since $c^{i} \geq 0$, if $X_{t}^{i}$ is generated by some $\left(c^{i}, \pi^{i}\right) \in \mathcal{A}_{i}$, we have $Z_{t}^{i} \geq \int_{0}^{t} \xi_{s} c_{s}^{i} d s \forall i \in C \cup U$, which implies that $Z_{t}^{i}$ is bounded below and it is therefore a supermartingale, by Fatou's lemma. Substituting the definition of $Z^{i}$ into the inequality $E\left(Z_{T}^{i}\right.$ $\left.\mathcal{F}_{t}\right) \leq Z_{t}^{i}$ and rearranging, we then see that

$$
\xi_{t} X_{t}^{i} \geq E\left(\xi_{T} X_{T}^{i}+\int_{t}^{T} \xi_{s}\left(c_{s}^{i} \Leftrightarrow e_{s}^{i}\right) d s \mid \mathcal{F}_{t}\right)
$$

$\forall i \in C \cup U$, which proves the claim.

Proof of Lemma 2: It is clear that nonsatiability implies $X_{T}^{i}=0$ a.s. Now, assume that the inequality (72) is met strictly in some set with positive probability. Then there exists a positive and adapted process $\varepsilon_{t}$ such that $E\left(\int_{t}^{T} \xi_{s}\left(c_{s}^{i}+\varepsilon_{s} \Leftrightarrow e_{s}^{i}\right) d s\right)=\xi_{t} X_{t}^{i}$. Denote by $X_{t}^{i, \varepsilon}$ the solution of (11) just substituting the process $c+\varepsilon$ for $c$. By lemma 1 we have $X_{t}^{i} \leq X_{t}^{i, \varepsilon}$, and consequently since $X_{t}^{i}$ satisfies (11) so does $X_{t}^{i, \varepsilon}$. This implies that $(\pi, c+\varepsilon)$ is admissible and furthermore $E\left(\int_{0}^{T} u\left(c_{t}+\varepsilon_{t}, t\right) d t\right)>E\left(\int_{0}^{T} u\left(c_{t}, t\right) d t\right)$, which contradicts the assumed optimality of $c$.

Proof of Proposition 3: The proof consists of showing that if a pair $\left(\pi^{\prime}, c^{\prime}\right)$ is admissible (i.e. satisfies (11)), then $c^{\prime}$ satisfies (16) and conversely, for any consumption policy $c^{\prime}$ that satisfies (16), there exists an adapted process $\pi$ such that $(\pi, c)$ is admissible. This will prove the first claim. The direct implication is immediate in view of (11), (12), wealth and (16). For the reverse implication, define $\pi$ as in (17). Certainly $\pi$ thus defined is progressively measurable. Substituting $\pi$ into (10) and applying Itô's lemma to $\xi_{t} X_{t}^{i}$, integrating and taking expectations leads to

$$
\xi_{t} X_{t}^{i}=X_{0}^{i}+\int_{0}^{t} \xi_{s}\left(e_{s}^{i} \Leftrightarrow c_{s}^{i}\right) d s+M_{t}=E\left(\int_{t}^{T} \xi_{s}\left(c_{s}^{i} \Leftrightarrow e_{s}^{i}\right) d s \mid \mathcal{F}_{t}\right)
$$

where in the first equality $M_{t}$ is the process defined in (18) and the second equality is obtained by using the static budget constraint $X_{0}^{i}+E\left(\int_{0}^{T} \xi_{s}\left(e_{s}^{i} \Leftrightarrow c_{s}^{i}\right) d s\right)=0$. Since $E\left(\int_{t}^{T} \xi_{s}\left(c_{s}^{i} \Leftrightarrow e_{s}^{i}\right) d s \mid \mathcal{F}_{t}\right) \geq$ 0 we conclude that $X_{t}^{i} \geq 0 \forall i \in C \cup U$. This establishes that $(\pi, c)$ is admissible.

Proof of Theorem 4: Let $c^{\prime}$ be an alternative feasible policy satisfying (16). Concavity of the utility function implies $u\left(c_{t}, t\right) \geq u\left(c_{t}^{\prime}, t\right)+u_{c}\left(c_{t}, t\right)\left(c_{t} \Leftrightarrow c_{t}^{\prime}\right)$ so that by $(20), u\left(c_{t}, t\right) \geq u\left(c_{t}^{\prime}, t\right)+$ $\left(y \Leftrightarrow \gamma_{t}\right) \xi_{t}\left(c_{t} \Leftrightarrow c_{t}^{\prime}\right)$. Integrating and taking expectations gives

$$
E\left(\int_{0}^{T} u\left(c_{t}, t\right)\right) \geq E\left(\int_{0}^{T} u\left(c_{t}^{\prime}, t\right)\right)+E\left(\int_{0}^{T}\left(y \Leftrightarrow \gamma_{t}\right) \xi_{t}\left(c_{t} \Leftrightarrow c_{t}^{\prime}\right) d t\right)
$$

Defining $g_{t} \equiv \int_{0}^{t} \xi_{s}\left(c_{s} \Leftrightarrow c_{s}^{\prime}\right) d s$ and integrating by parts we get

$$
\int_{0}^{T} \gamma_{t} d g_{t}=\Leftrightarrow \int_{0}^{T} g_{t} d \gamma_{t}+\gamma_{T} g_{T}=\Leftrightarrow \int_{0}^{T} \int_{0}^{t} \xi_{s}\left(c_{s} \Leftrightarrow c_{s}^{\prime}\right) d s d \gamma_{t}+\gamma_{T} \int_{0}^{T} \xi_{t}\left(c_{t} \Leftrightarrow c_{t}^{\prime}\right) d t .
$$

Taking expectations on each side of (74) and using the definition of $g_{t}$, we can write

$$
E\left(\int_{0}^{T} \gamma_{t} \xi_{t}\left(c_{t} \Leftrightarrow c_{t}^{\prime}\right) d t\right)=E\left(\int_{0}^{T}\left(\int_{0}^{T} \xi_{t}\left(c_{t} \Leftrightarrow c_{t}^{\prime}\right) d t \Leftrightarrow \int_{0}^{t} \xi_{s}\left(c_{s} \Leftrightarrow c_{s}^{\prime}\right) d s\right) d \gamma_{t}\right)
$$




$$
\begin{aligned}
& =E\left(\int_{0}^{T}\left(\int_{t}^{T} \xi_{s}\left(c_{s} \Leftrightarrow c_{s}^{\prime}\right) d s\right) d \gamma_{t}\right) \\
& =E\left(\int_{0}^{T} E\left(\int_{t}^{T} \xi_{s}\left(c_{s} \Leftrightarrow c_{s}^{\prime}\right) d s \mid \mathcal{F}_{t}\right) d \gamma_{t}\right) \\
& =E\left(\int_{0}^{T} \xi_{t}\left(X_{t} \Leftrightarrow X_{t}^{\prime}\right) d \gamma_{t}\right)
\end{aligned}
$$

and substituting this into (73), we arrive at

$$
E\left(\int_{0}^{T} u\left(c_{t}, t\right)\right) \geq E\left(\int_{0}^{T} u\left(c_{t}^{\prime}, t\right)\right)+y E\left(\int_{0}^{T} \xi_{t}\left(c_{t} \Leftrightarrow c_{t}^{\prime}\right) d t\right) \Leftrightarrow E\left(\int_{0}^{T} \xi_{t}\left(X_{t} \Leftrightarrow X_{t}^{\prime}\right) d \gamma_{t}\right)
$$

Now, by the second condition in (20) and the feasibility of $c$ and $c^{\prime}$ we have $E\left(\int_{0}^{T} \xi_{t}\left(c_{t} \Leftrightarrow c_{t}^{\prime}\right) d t\right)$ $\geq 0$. Also, the complementary slackness condition in (20) implies that $E\left(\int_{0}^{T} \xi_{t} X_{t} d \gamma_{t}\right)=0$. But since $X_{t}^{\prime} \geq 0$ ( $c^{\prime}$ satisfies (16) by hypothesis), and also since $\gamma_{t}$ is nondecreasing, we conclude that $U(c) \geq U\left(c^{\prime}\right)$ for some $c$ satisfying (20) and any $c^{\prime}$ satisfying (16).

Proof of Lemma 5: Consider the map $F_{t}(y, \cdot)$ for $y>0$ fixed. Note that $F_{t}(y, \cdot):[0, y] \rightarrow$ $\left[F_{t}(y, 0), \infty\right)$ is continuous and strictly increasing. Thus a unique inverse exists and if 0 is in the range of $F_{t}(y, \cdot)$ the inverse $F_{t}^{-1}(y, 0)$ is well defined. It follows that

$$
\Delta_{t}(y)=F_{t}^{-1}(y, 0) 1_{\left\{F_{t}(y, 0)<0\right\}}
$$

is uniquely defined and satisfies (23)-(24) for all $t \in[0, T]$. Furthermore $\Delta_{t}(y)$ is a continuous and nondecreasing function of $y$. The map $E \int_{0}^{T} \xi_{s}\left[I\left(\left(y \Leftrightarrow \Delta_{0, s}^{*}(y)\right) \xi_{s}\right) \Leftrightarrow e_{s}\right] d s$ is then continuous with respect to $y$ and tends to $\infty$ as $y \rightarrow 0$. Taking $y^{*}=\inf \left\{y>0: E \int_{0}^{T} \xi_{s}\left[I\left(\left(y \Leftrightarrow \Delta_{0, s}^{*}(y)\right) \xi_{s}\right) \Leftrightarrow e_{s}\right] d s=\right.$ $\left.n S_{0}\right\}$ ensures that the initial condition (27) is satisfied and $\Delta_{0}=0$.

Proof of Proposition 8: (i) Consider the example of Figure 1. For the numerical values selected the maximal value of the function $\left(\frac{1}{2} \Leftrightarrow \frac{1}{R}\right) \theta^{2}$ when $R \in[.02,3]$ is .015 . Since $r=.1$ we have $r>\left(\frac{1}{2} \Leftrightarrow \frac{1}{R}\right) \theta^{2}$. This ensures that unconstrained consumption is an increasing function of relative risk aversion. The numerical simulation shows that constrained consumption is humped.

(ii) The optimal liquid wealth process associated with the initial conditions $\left(k X_{0}, k e_{0}\right)$ is

$$
X_{t}^{k}=E\left[\int_{t}^{T}\left[\xi_{t, s}^{1-1 / R}\left(y^{k} \Leftrightarrow \gamma_{s}^{k}\right)^{-1 / R} \Leftrightarrow \xi_{s} k e_{s}\right] d s \mid \mathcal{F}_{t}\right]
$$

It is easy to verify that $X_{t}^{k}=k X_{t}, y^{k}=k^{-R} y$ and $\gamma_{s}^{k}=k^{-R} \gamma_{s}$ satisfies the required constraints. Homogeneity of the consumption function follows.

Proof of Lemma 7: Define the process $N_{t}=E\left(\int_{t}^{T} \xi_{s}\left(c_{s} \Leftrightarrow e_{s}\right) d s \mid \mathcal{F}_{t}\right)$ evaluated at optimal consumption. Since optimal consumption satisfies $N_{t} \geq 0$ for all $t \in[0, T]$ it must be the case that $d N_{t} \geq 0$ on the event $\left\{N_{t}=0\right\}$. Thus $d[N]_{t}=0$ on $\left\{N_{t}=0\right\}$ where [N] represents the quadratic variation of $N$. Consider now the process $M$ defined in Proposition 3. We have

$$
M_{t} \equiv E\left(\int_{0}^{T} \xi_{s}\left(c_{s} \Leftrightarrow e_{s}\right) d s \mid \mathcal{F}_{t}\right) \Leftrightarrow E\left(\int_{0}^{T} \xi_{s}\left(c_{s} \Leftrightarrow e_{s}\right) d s\right)=N_{t} \Leftrightarrow \int_{0}^{t} \xi_{s}\left(c_{s} \Leftrightarrow e_{s}\right) d s \Leftrightarrow N_{0} .
$$

Integrating the quadratic variation over $\left\{N_{t}=0\right\}$ and using $d[N]_{t}=0$ over that event leads to

$$
E \int_{0}^{T} 1_{\left\{N_{t}=0\right\}} d[M]_{t}=E \int_{0}^{T} 1_{\left\{N_{t}=0\right\}} d[N]_{t}=0 .
$$


But Proposition 3 implies $d[M]_{t}=\left(\Leftrightarrow X_{t} \xi_{t} \theta_{t}+\pi_{t} \sigma_{t}\right)^{2} d t$. Using $X_{t}=0$ on $\left\{N_{t}=0\right\}$ and substituting yields

$$
E \int_{0}^{T} 1_{\left\{N_{t}=0\right\}}\left(\pi_{t} \sigma_{t}\right)^{2} d t=0
$$

We conclude that $\pi_{t}=0$ on $\left\{N_{t}=0\right\}$.

Proof of Theorem 6: Recall the functions $g_{t}$ and $h_{t}$ such that

$$
\begin{aligned}
C_{t} & =I_{1}\left(g_{t}(x)\right)+I_{2}\left(x g_{t}(x)\right) \\
h_{t}(x) & =g_{t}(x)\left[I_{2}\left(x g_{t}(x)\right) \Leftrightarrow e_{t}^{2}\right] .
\end{aligned}
$$

These functions have the following properties

Lemma 17 For all $t \in[0, T]$ the function $g_{t}(\cdot):[0, y] \rightarrow\left[g_{t}(y), \infty\right)$ satisfies

(i) $g_{t}(\cdot)$ is strictly decreasing

(ii) $\lim _{x \rightarrow 0} g_{t}(x)=\infty$

(iii) $\lim _{x \rightarrow y} g_{t}(x)=g_{t}(y)>0$

(iv) $g_{t}^{\prime}(x)=\Leftrightarrow \frac{I_{2}^{\prime}\left(x g_{t}(x)\right) g_{t}(x)}{I_{1}^{\prime}\left(g_{t}(x)\right)+I_{2}^{\prime}\left(x g_{t}(x)\right) x}$.

Proof of Lemma 17: The function $g_{t}(\cdot)$ is continuously differentiable on $[0, y]$. An application of the Implicit Function Theorem yields (iv). Property (i) follows from (iv). When $x \rightarrow 0$ we have $I_{2}\left(x g_{t}(x)\right) \rightarrow \infty$ for all $g_{t}(x)$ finite. Since the left hand side of $(75)$ is finite and $I_{1}(\cdot)$ is nonnegative the limit (ii) holds. Property (iii) is straightforward.

Lemma 18 For all $t \in[0, T]$ the function $h_{t}(\cdot):[0, y] \rightarrow\left[h_{t}(y), 0\right]$ satisfies

(i) $\exists x^{*} \in(0, y)$ such that $h_{t}(x)$ is strictly decreasing for $x<x^{*}$

(ii) $\lim _{x \rightarrow 0} h_{t}(x)=\infty$

(iii) $\lim _{x \rightarrow y} h_{t}(x)=h_{t}(y)>(<) 0 \Leftrightarrow I_{2}\left(y g_{t}(y)\right) \Leftrightarrow e_{t}^{2}>(<) 0$.

(iv) $h_{t}^{\prime}(x)=g_{t}^{\prime}(x)\left[I_{2}\left(x g_{t}(x)\right) \Leftrightarrow e_{t}^{2}\right]+g_{t}(x) I_{2}^{\prime}\left(x g_{t}(x)\right)\left[g_{t}(x)+x g_{t}^{\prime}(x)\right]$.

Proof of Lemma 18: Properties (ii), (iii) and (iv) are straightforward. To prove (i) note that (75) implies $I_{2}\left(x g_{t}(x)\right) \Leftrightarrow e_{t}^{2} \geq 0$ for $x$ sufficiently low. Let $x^{*}$ be such that $I_{2}\left(x^{*} g_{t}\left(x^{*}\right)\right) \Leftrightarrow e_{t}^{2}=0$ or if no such $x^{*}$ exists in $(0, y]$ set $x^{*}=y$. Property (iv) of Lemma 17 implies

$$
g_{t}(x)+x g_{t}^{\prime}(x)=\frac{I_{1}^{\prime}\left(g_{t}(x)\right) g_{t}(x)}{I_{1}^{\prime}\left(g_{t}(x)\right)+I_{2}^{\prime}\left(x g_{t}(x)\right) x}>0 .
$$

Hence $I_{2}\left(x g_{t}(x)\right) \Leftrightarrow e_{t}^{2}$ is strictly decreasing and for $x<x^{*}$ we have $I_{2}\left(x g_{t}(x)\right) \Leftrightarrow e_{t}^{2}>0$. Since $g_{t}^{\prime}(x)<0$ it then follows from (iv) that $h_{t}^{\prime}(x)<0$ for $x<x^{*}$.

Consider now the process

$$
F_{t}\left(y, \Delta_{t}\right)=E\left(\int_{t}^{T} h_{s}\left(y \Leftrightarrow \Delta_{t, s}^{*}\right) d s \mid \mathcal{F}_{t}\right), t \in[0, T] .
$$

Solving

$$
\begin{gathered}
F_{t}\left(y, \Delta_{t}\right) \geq 0, \quad t \in[0, T] \\
F_{t}\left(y, \Delta_{t}\right) d \Delta_{t} \leq 0, \quad t \in[0, T]
\end{gathered}
$$


is identical to the problem that we solved before. As long as the integrand $h_{t}(x)$ has the right properties we can apply the construction in Lemma 5 and Theorem 6 . Since the function $h_{t}(y \Leftrightarrow$ $\left.\Delta_{t, s}^{*}\right)$ is strictly increasing with respect to $\Delta_{t}$ for $\Delta_{t}$ sufficiently large, we can always find $\Delta_{t}(y)$ such that $F_{t}\left(y, \Delta_{t}(y)\right) \geq 0$. Hence the same construction as in the case of exogenous state price density works. The same arguments also lead to the multiplier $y$ which satisfies the static budget constraint (25) of the constrained agent in equilibrium.

Proof of Proposition 11: From (41) and the definition of $H$ we get the expression

$$
\frac{1}{R_{t}^{a}}=\Leftrightarrow \frac{U^{\prime}}{C U^{\prime \prime}}=\Leftrightarrow \frac{H(C)}{C H^{\prime}(C)}=\Leftrightarrow \frac{H(C)}{C} \sum_{i} \frac{I_{i}^{\prime}}{\alpha_{t}^{i}}=\Leftrightarrow \sum_{i} \frac{\left(y^{i} \Leftrightarrow \gamma_{t}^{i}\right) H(C)}{u_{i}^{\prime \prime} C}=\Leftrightarrow \sum_{i} \frac{u_{i}^{\prime}}{u_{i}^{\prime \prime} C}=\sum_{i} \frac{1}{R_{t}^{i}} \frac{c_{i}}{C}
$$

for the inverse of the aggregator's relative risk aversion. Note that we used the first order conditions of the individual agents' problem to obtain the last equality.

Proof of Theorem 12: To simplify notation we assume that the unconstrained agent has a liquidity constraint multiplier $\gamma^{1}=0$. Both agents have utility function $u(c, t)=\rho_{t}^{-1} u(c)$ where $\rho_{t}=\exp (\beta t)$; let $I(y)=u_{c}^{-1}(y)$ denote the inverse of $u_{c}$. The goods market clearing condition is $C_{t}=\sum_{i} I_{i}\left(\left(y^{i} \Leftrightarrow \gamma_{t}^{i}\right) \rho_{t} \xi_{t}\right)$. Applying Ito's lemma on both sides of this equation yields

$$
d C_{t}=\sum_{i} I_{i}^{\prime} \times\left(y^{i} \Leftrightarrow \gamma_{t}^{i}\right) \rho_{t} \xi_{t}\left[\frac{d \rho_{t}}{\rho_{t}}+\frac{d \xi_{t}}{\xi_{t}} \Leftrightarrow \frac{d \gamma_{t}^{i}}{y^{i} \Leftrightarrow \gamma_{t}^{i}}\right]+\frac{1}{2} \sum_{i} I_{i}^{\prime \prime} \times\left(\left(y^{i} \Leftrightarrow \gamma_{t}^{i}\right) \rho_{t} \xi_{t}\right)^{2} \frac{d[\xi]_{t}}{\left(\xi_{t}\right)^{2}}
$$

where $[\xi]_{t}$ denotes the quadratic variation process. Dividing by $C$

$$
\frac{d C_{t}}{C_{t}}=\Leftrightarrow \frac{1}{R_{t}^{a}}\left[\frac{d \rho_{t}}{\rho_{t}}+\frac{d \xi_{t}}{\xi_{t}}\right]+\frac{1}{R_{t}^{2}} \frac{c_{t}^{2}}{C_{t}} \frac{d \gamma_{t}}{y \Leftrightarrow \gamma_{t}}+\frac{1}{2} \frac{P_{t}^{a}}{\left(R_{t}^{a}\right)^{2}} \frac{d[\xi] t}{\left(\xi_{t}\right)^{2}}
$$

where

$$
\begin{aligned}
R_{t}^{a} & =\Leftrightarrow\left[\frac{\sum_{i} I_{i}^{\prime}\left(\left(y^{i} \Leftrightarrow \gamma_{t}^{i}\right) \rho_{t} \xi_{t}\right)\left(y^{i} \Leftrightarrow \gamma_{t}^{i}\right) \rho_{t} \xi_{t}}{\sum_{i} I_{i}\left(\left(y^{i} \Leftrightarrow \gamma_{t}^{i}\right) \rho_{t} \xi_{t}\right)}\right]^{-1} \\
P_{t}^{a} & =\left(R_{t}^{a}\right)^{2} \frac{\sum_{i} I_{i}^{\prime \prime}\left(\left(y^{i} \Leftrightarrow \gamma_{t}^{i}\right) \rho_{t} \xi_{t}\right)\left(\left(y^{i} \Leftrightarrow \gamma_{t}^{i}\right) \rho_{t} \xi_{t}\right)^{2}}{\sum_{i} I_{i}\left(\left(y^{i} \Leftrightarrow \gamma_{t}^{i}\right) \rho_{t} \xi_{t}\right)} .
\end{aligned}
$$

and $R_{t}^{2}$ is the coefficient of risk aversion of the constrained agent. By definition $I_{i}(y)$ satisfies $u_{c}\left(I_{i}(y)\right)=y$. Substituting $d \rho_{t}=\rho_{t} \beta d t$ and $d \xi_{t}=\Leftrightarrow \xi_{t}\left[d R_{t}+\theta_{t} d W_{t}\right]$ yields the equilibrium formulas $\theta_{t}=R_{t}^{a} \sigma^{c}$ and

$$
d R_{t}=\left(\beta+R_{t}^{a} \mu^{c} \Leftrightarrow \frac{1}{2} R_{t}^{a} P_{t}^{a}\left(\sigma^{c}\right)^{2}\right) d t \Leftrightarrow \frac{R_{t}^{a}}{R_{t}^{2}} \frac{c_{t}^{2}}{C_{t}} \frac{d \gamma_{t}}{y \Leftrightarrow \gamma_{t}} .
$$

The formulas in the Theorem follow from the definitions $\theta_{t}=\sigma_{t}^{-1}\left(\mu_{t} \Leftrightarrow r_{t}\right)$ and $d R_{t}=r_{t} d t+d A_{t}$.

Proof of Proposition 10: Construct the positive process $\alpha_{t}^{i}=1 /\left(y^{i} \Leftrightarrow \gamma_{t}^{i}\right)$. For any feasible allocation $\left\{x_{t}^{i}\right\}$ we have

$$
E \int_{0}^{T} \sum_{i} \alpha_{t}^{i} u^{i}\left(c_{t}^{i}, t\right) d t \Leftrightarrow E \int_{0}^{T} \sum_{i} \alpha_{t}^{i} u^{i}\left(x_{t}^{i}, t\right) d t \geq E \int_{0}^{T} \sum_{i} \alpha_{t}^{i}\left(y^{i} \Leftrightarrow \gamma_{t}^{i}\right) \xi_{t}\left(c_{t}^{i} \Leftrightarrow x_{t}^{i}\right) d t \geq 0
$$

where the first inequality follows from concavity and the first order conditions, the second inequality follows from the definition of the process $\alpha_{t}^{i}$ and the feasibility of the allocations $\left\{c_{t}^{i}\right\}$ 
and $\left\{x_{t}^{i}\right\}$. This inequality shows that $\left\{c_{t}^{i}\right\}$ solves (39). To prove that $\left(\left\{D_{t}+e_{t}, 1\right\}\right)$ is a no trade equilibrium for the single agent economy, we just need to show that $\left\{C_{t}\right\}$ solves

$$
\sup _{x} U(x) \quad \text { s.t. } \quad E\left(\int_{0}^{T} \xi_{t}\left(x_{t} \Leftrightarrow C_{t}\right) d t\right) \leq 0 .
$$

Suppose that there exists a process $x$ such that $U(x)>U(C)$ and $E\left(\int_{0}^{T} \xi_{t}\left(x_{t} \Leftrightarrow C_{t}\right) d t\right) \leq 0$. This implies that there exists a process $\left\{x_{t}^{i}\right\}$ such that $\sum_{i} x_{t}^{i}=x_{t}$ and, using $\sum_{i} c_{t}^{i}=C_{t}$, we have

$$
E \sum_{i} \int_{0}^{T}\left(\alpha_{t}^{i} u^{i}\left(x_{t}^{i}, t\right) \Leftrightarrow \xi_{t}\left(x_{t}^{i} \Leftrightarrow e_{t}^{i}\right)\right) d t>E \sum_{i} \int_{0}^{T}\left(\alpha_{t}^{i} u^{i}\left(c_{t}^{i}, t\right) \Leftrightarrow \xi_{t}\left(c_{t}^{i} \Leftrightarrow e_{t}^{i}\right)\right) d t
$$

but this contradicts the fact that $\frac{\partial u^{i}\left(c_{t}^{i}, t\right)}{\partial c_{t}^{i}}=\frac{1}{\alpha_{t}^{i}} \xi_{t}$.

Proof of Theorem 15: Suppose that a competitive equilibrium exists with $\sigma_{1} \neq 0$. Recall that individual SPDs are,

$$
\xi_{t}^{i}=b_{t} \exp \left[\Leftrightarrow \int_{0}^{t} \theta_{1 v}^{i} d W_{1 v} \Leftrightarrow \int_{0}^{t} \theta_{2 v}^{i} d W_{2 v} \Leftrightarrow \frac{1}{2} \int_{0}^{t}\left[\left(\theta_{1 v}^{i}\right)^{2}+\left(\theta_{2 v}^{i}\right)^{2}\right] d v\right],
$$

for processes $\theta^{i} \equiv\left(\theta_{1}^{i}, \theta_{2}^{i}\right), i=1,2$, defined as

$$
\theta_{t}^{i}=\left[\begin{array}{l}
\theta_{1 t}^{i} \\
\theta_{2 t}^{i}
\end{array}\right] \equiv\left[\begin{array}{ll}
\sigma_{1 t} & \sigma_{2 t} \\
0 & 1
\end{array}\right]^{-1}\left[\begin{array}{l}
\mu_{t} \Leftrightarrow r_{t} \\
\alpha_{t}^{i} \Leftrightarrow r_{t}
\end{array}\right]=\frac{1}{\sigma_{1 t}}\left[\begin{array}{l}
\mu_{t} \Leftrightarrow r_{t} \Leftrightarrow \sigma_{2 t} \theta_{2 t}^{i} \\
\sigma_{1 t}\left(\alpha_{t}^{i} \Leftrightarrow r_{t}\right)
\end{array}\right] .
$$

Our first lemma expresses the competitive equilibrium in terms of individual market prices of risk.

Lemma 19 In equilibrium the interest rate and the individual prices of risk satisfy

$$
\begin{gathered}
d R_{t}=\left(\Leftrightarrow \frac{1}{A_{t}} \mu^{c}+\frac{1}{2} \sum_{i}\left[\frac{B_{t}^{i}}{A_{t}}\right]\left[\left(\theta_{1 t}^{i}\right)^{2}+\left(\theta_{2 t}^{i}\right)^{2}\right]\right) d t \Leftrightarrow \frac{A_{t}^{2}}{A_{t}} \frac{1}{y \Leftrightarrow \gamma_{t}} d \gamma_{t} \\
\sum_{i} A_{t}^{i} \theta_{1 t}^{i}=\Leftrightarrow \sigma_{t}^{c} \\
\sum_{i} A_{t}^{i} \theta_{2 t}^{i}=0
\end{gathered}
$$

where

$$
A_{t}^{i}=\frac{I_{i}^{\prime}\left(y_{i} \xi_{t}^{i}\right) y_{i} \xi_{t}^{i}}{\sum_{i} I_{i}\left(y_{i} \xi_{t}^{i}\right)} \quad \text { and } \quad B_{t}^{i}=\frac{I_{i}^{\prime \prime}\left(y_{i} \xi_{t}^{i}\right)\left(y_{i} \xi_{t}^{i}\right)^{2}}{\sum_{i} I_{i}\left(y_{i} \xi_{t}^{i}\right)}
$$

and $A_{t}=A_{t}^{1}+A_{t}^{2}$ where $y_{1}=1$ and $y_{2}=y \Leftrightarrow \gamma_{t}$.

Proof of Lemma 19: Ito's lemma along with the goods market equilibrium condition $C_{t}=$ $I_{1}\left(\xi_{t}^{1}\right)+I_{2}\left(\xi_{t}^{1}\left(y \Leftrightarrow \gamma_{t}\right) \eta_{t}\right)$ gives (to simplify notation we omit the arguments of the functions $I_{i}(\cdot)$ )

$$
\begin{aligned}
C_{t}\left[\mu^{c} d t+\sigma_{t}^{c} d W_{1 t}\right]= & \Leftrightarrow I_{1}^{\prime} \xi_{t}^{1}\left[d R_{t}+\theta_{1 t}^{1} d W_{1 t}+\theta_{2 t}^{1} d W_{2 t}\right] \\
& \Leftrightarrow I_{2}^{\prime} \xi_{t}^{1} \eta_{t}\left[\left(y \Leftrightarrow \gamma_{t}\right)\left[d R_{t}+\theta_{1 t}^{2} d W_{1 t}+\theta_{2 t}^{2} d W_{2 t}\right]+d \gamma_{t}\right] \\
& +\frac{1}{2}\left[I_{1}^{\prime \prime} \times\left(\xi_{t}^{1}\right)^{2}\left\|\theta_{t}^{1}\right\|^{2}+I_{2}^{\prime \prime} \times\left(\left(y \Leftrightarrow \gamma_{t}\right) \xi_{t}^{2}\right)^{2}\left\|\theta_{t}^{2}\right\|^{2}\right] d t
\end{aligned}
$$

where $\left\|\theta_{t}^{i}\right\|^{2}=\left(\theta_{1 t}^{i}\right)^{2}+\left(\theta_{2 t}^{i}\right)^{2}, i=1,2$. Collecting terms and setting the coefficients of $d W_{1 t}, d W_{2 t}$ and of the bounded variation terms equal to zero yields the formulas in the lemma. 
Lemma 20 In equilibrium the interest rate and the Sharpe ratio satisfy

$$
\begin{gathered}
d R_{t}=\left(\Leftrightarrow \frac{1}{A_{t}} \mu^{c}+\frac{1}{2}\left[\frac{B_{t}}{A_{t}^{3}}\right]\left(\sigma_{t}^{c}\right)^{2}+\frac{1}{2} \frac{\sigma_{t}^{2}}{\sigma_{1 t}^{2}} \sum_{i}\left[\frac{B_{t}^{i}}{A_{t}}\left(\theta_{2 t}^{i}\right)^{2}\right]+\frac{1}{A_{t}} \frac{\sigma_{2 t} \sigma_{t}^{c}}{\sigma_{1 t}} \sum_{i}\left[\frac{B_{t}^{i}}{A_{t}} \theta_{2 t}^{i}\right]\right) d t \Leftrightarrow \frac{A_{t}^{2}}{A_{t}} \frac{d \gamma_{t}}{y \Leftrightarrow \gamma_{t}} \\
\frac{\mu_{t} \Leftrightarrow r_{t}}{\sigma_{1 t}}=\Leftrightarrow \frac{1}{A_{t}} \sigma_{t}^{c}
\end{gathered}
$$

where $\sigma_{t}^{2}=\sigma_{1 t}^{2}+\sigma_{2 t}^{2}$ and $B_{t}=\sum_{i} B_{t}^{i}$.

Proof of Lemma 20: Multiplying both sides of (82)-(83) by

$$
\left[\begin{array}{ll}
\sigma_{1 t} & \sigma_{2 t} \\
0 & 1
\end{array}\right]
$$

leads to the formula for the Sharpe ratio. Using the definitions of the market prices of risk yields

$$
\left\|\theta_{t}^{i}\right\|^{2}=\left[\frac{1}{\sigma_{1 t}}\left(\mu_{t} \Leftrightarrow r_{t} \Leftrightarrow \sigma_{2 t} \theta_{2 t}^{i}\right)\right]^{2}+\left(\theta_{2 t}^{i}\right)^{2}=\left(\frac{\mu_{t} \Leftrightarrow r_{t}}{\sigma_{1 t}}\right)^{2}+\frac{\sigma_{1 t}^{2}+\sigma_{2 t}^{2}}{\sigma_{1 t}^{2}}\left(\theta_{2 t}^{i}\right)^{2} \Leftrightarrow 2\left(\frac{\mu_{t} \Leftrightarrow r_{t}}{\sigma_{1 t}}\right) \frac{\sigma_{2 t}}{\sigma_{1 t}} \theta_{2 t}^{i}
$$

for $i=1,2$, where $\left\|\theta_{t}^{i}\right\|^{2}=\left(\theta_{1 t}^{i}\right)^{2}+\left(\theta_{2 t}^{i}\right)^{2}$. Substituting in the interest formula (81) produces

$$
\begin{aligned}
d R_{t}= & \left(\Leftrightarrow \frac{1}{A_{t}} \mu^{c}+\frac{1}{2}\left[\frac{B_{t}}{A_{t}}\right]\left(\frac{\mu_{t} \Leftrightarrow r_{t}}{\sigma_{1 t}}\right)^{2}+\frac{1}{2} \frac{\sigma_{1 t}^{2}+\sigma_{2 t}^{2}}{\sigma_{1 t}^{2}} \sum_{i}\left[\frac{B_{t}^{i}}{A_{t}}\left(\theta_{2 t}^{i}\right)^{2}\right]\right) d t \\
& \Leftrightarrow\left(\frac{\mu_{t} \Leftrightarrow r_{t}}{\sigma_{1 t}}\right) \frac{\sigma_{2 t}}{\sigma_{1 t}} \sum_{i}\left[\frac{B_{t}^{i}}{A_{t}} \theta_{2 t}^{i}\right] d t \Leftrightarrow \frac{A_{t}^{2}}{A_{t}} \frac{d \gamma_{t}}{y \Leftrightarrow \gamma_{t}}
\end{aligned}
$$

Substituting the Sharpe ratio formula in this expression produces the result in the lemma.

Differentiating the first order condition $u^{\prime}\left(I_{i}\left(x^{i}\right)\right)=x^{i}$, where $x^{i}=\left(y^{i} \Leftrightarrow \gamma^{i}\right) \xi_{t}^{i}$ gives $1=$ $u^{\prime \prime}\left(I_{i}\left(x^{i}\right)\right) I_{i}^{\prime}\left(x^{i}\right)$ and $0=u^{\prime \prime \prime}\left(I_{i}\left(x^{i}\right)\right) I_{i}^{\prime}\left(x^{i}\right)^{2}+u^{\prime \prime}\left(I_{i}\left(x^{i}\right)\right) I_{i}^{\prime \prime}\left(x^{i}\right)$ so that

$$
I_{i}^{\prime}\left(x^{i}\right) x^{i}=\frac{u^{\prime}\left(I_{i}\left(x^{i}\right)\right)}{u^{\prime \prime}\left(I_{i}\left(x^{i}\right)\right)}=\Leftrightarrow \frac{c_{t}^{i}}{R_{t}^{i}} \quad \text { and } \quad I_{i}^{\prime \prime}\left(x^{i}\right)\left(x^{i}\right)^{2}=\Leftrightarrow \frac{u^{\prime \prime \prime}\left(I_{i}\left(x^{i}\right)\right)}{u^{\prime \prime}\left(I_{i}\left(x^{i}\right)\right)} \frac{u^{\prime}\left(I_{i}\left(x^{i}\right)\right)^{2}}{u^{\prime \prime}\left(I_{i}\left(x^{i}\right)\right)^{2}}=\frac{c_{t}^{i} P_{t}^{i}}{\left(R_{t}^{i}\right)^{2}}
$$

leading to $A_{t}^{i}=\Leftrightarrow c_{t}^{i} /\left(C_{t} R_{t}^{i}\right)$ and $B_{t}^{i}=c_{t}^{i} P_{t}^{i} /\left(C_{t}\left(R_{t}^{i}\right)^{2}\right)$. Substitution in the expressions of the lemma produces

$$
\begin{aligned}
d R_{t}= & \left(\frac{1}{\sum_{i} \frac{c_{t}^{i}}{R_{t}^{i}}} C_{t} \mu^{c} \Leftrightarrow \frac{1}{2}\left[\frac{\sum_{i} \frac{c_{t}^{i} P_{t}^{i}}{\left(\sum_{t}^{i}\right)^{2}}}{\left(\sum_{t}^{i}\right)_{t}^{3}}\right]\left(C_{t} \sigma_{t}^{c}\right)^{2} \Leftrightarrow \frac{1}{2} \frac{1}{\sum_{i} \frac{c_{t}^{i}}{R_{t}^{i}}} \frac{\sigma_{1 t}^{2}+\sigma_{2 t}^{2}}{\sigma_{1 t}^{2}} \sum_{i}\left[\frac{c_{t}^{i} P_{t}^{i}}{\left(R_{t}^{i}\right)^{2}}\left(\theta_{2 t}^{i}\right)^{2}\right]\right) d t \\
& +\frac{1}{\left(\sum_{i} \frac{c_{t}^{i}}{R_{t}^{i}}\right)^{2}} C_{t} \frac{\sigma_{2 t} \sigma_{t}^{c}}{\sigma_{1 t}} \sum_{i}\left[\frac{c_{t}^{i} P_{t}^{i}}{\left(R_{t}^{i}\right)^{2}} \theta_{2 t}^{i}\right] d t \Leftrightarrow \frac{\frac{c_{t}^{2}}{R_{t}^{2}}}{\sum_{i} \frac{c_{t}^{i}}{R_{t}^{i}} \frac{d \gamma_{t}}{y \Leftrightarrow \gamma_{t}}}
\end{aligned}
$$

and $\left(\mu_{t} \Leftrightarrow r_{t}\right) / \sigma_{1 t}=\left[\sum_{i}\left(c_{t}^{i} / R_{t}^{i}\right)\right]^{-1} C_{t} \sigma_{t}^{c}$. This completes the proof of Theorem 15 .

Proof of Corollary16: Suppose that $R_{t}^{1}=R_{t}^{2}=R$. Then $P_{t}^{i}=1+R$ and

$$
\begin{aligned}
d R_{t}= & \left(R \mu^{c} \Leftrightarrow \frac{1}{2} R(1+R)\left(\sigma_{t}^{c}\right)^{2} \Leftrightarrow \frac{1}{2} \frac{(1+R)}{R} \frac{\left(\sigma_{1 t}^{2}+\sigma_{2 t}^{2}\right)}{\sigma_{1 t}^{2}} \sum_{i}\left[\frac{c_{t}^{i}}{C_{t}}\left(\theta_{2 t}^{i}\right)^{2}\right]\right) d t \\
& +(1+R) \sigma_{t}^{c} \frac{\sigma_{2 t}}{\sigma_{1 t}} \sum_{i}\left[\frac{c_{t}^{i}}{C_{t}} \theta_{2 t}^{i}\right] d t \Leftrightarrow \frac{c_{t}^{2}}{C_{t}} \frac{d \gamma_{t}}{y \Leftrightarrow \gamma_{t}}
\end{aligned}
$$

while $\left(\mu_{t} \Leftrightarrow r_{t}\right) / \sigma_{1 t}=R \sigma_{t}^{c}$. But we also have $0=\sum_{i} A_{t}^{i} \theta_{2 t}^{i}=\Leftrightarrow \frac{1}{R} \sum_{i}\left(c_{t}^{i} / C_{t}\right) \theta_{2 t}^{i}$. Substituting gives the expression in the Corollary. 


\subsection{Appendix B: a formulation of the lagrangian problem.}

Let $\mathcal{H}$ be the set of nonnegative, continuous, adapted and bounded processes, and let $\mathcal{L}_{\infty}$ be the space of essentially bounded, continuous and adapted processes. Assume $e, \xi \in \mathcal{H}$. The optimization problem can be formulated as

$$
\begin{aligned}
J & =\sup _{c \in \mathcal{H}} U(c) \text { s.t. } \\
\mathcal{A}(c) & \equiv E\left(\int_{0}^{T} \xi_{t}\left(c_{t} \Leftrightarrow e_{t}\right) d t\right) \Leftrightarrow X_{0} \leq 0 \\
\mathcal{V}(c) & \equiv E\left(\int_{t}^{T} \xi_{s}\left(c_{s} \Leftrightarrow e_{s}\right) d s \mid \mathcal{F}_{t}\right) \geq 0
\end{aligned}
$$

where $\mathcal{V}: \mathcal{H} \rightarrow \mathcal{L}_{\infty}$. Denote by $b a$ the set of bounded additive set functions, i.e. the dual space of $\mathcal{L}_{\infty}$ (Dunford and Schwartz (1958), IV.6), and let $b a^{\oplus}$ be the positive cone of $b a$. Since $\mathcal{H}$ is a linear vector space, $\mathcal{L}_{\infty}$ is a normed vector space whose positive cone has a nonempty interior, $U$ is concave, $\mathcal{A}$ and $\mathcal{V}$ are convex and $J$ is finite, the saddle point theorem (i.e. Luenberger (1969), Theorem 8.1 and Corollary 1) gives

$$
J=\sup _{c \in \mathcal{H}} \inf _{z \in b a \oplus, \lambda \in \mathrm{R}_{+}} U(c)+\langle\mathcal{V}(c), z\rangle+\mathcal{A}(c) \lambda
$$

where $\langle a, b\rangle$ is the value of the functional $b$ evaluated at $a$. Now from Yosida and Hewitt ((1952), 1.23 and 2.3) it follows that $\forall z \in b a^{\oplus} \forall x \in \mathcal{L}_{\infty}$, there exist a nonnegative countably additive measure $\mu^{m}$ and pure charge (or a nonnegative purely finitely additive set function) $\mu^{c h}$ such that

$$
\langle\mathcal{V}(c), z\rangle=\int_{\Omega \times[0, T]} \mathcal{V}(c) d \mu^{m}+\int_{\Omega \times[0, T]} \mathcal{V}(c) d \mu^{c h}
$$

$>$ From Yosida and Hewitt $(1952,1.22)$, there exists a sequence of predictable sets $\left\{S_{i}\right\}$ such that $\lim _{i \uparrow \infty} P \times \operatorname{Leb}\left(S_{i}\right)=0, \lim _{i \uparrow \infty} \mu^{m}\left(S_{i}\right)=0$ and $\mu^{c h}\left(S_{i}^{c}\right)=0 \forall i$. Now construct the decreasing sequence of predictable sets $A_{i}=\bigcap_{n=1}^{i} S_{n}$. Clearly, $\lim _{i \uparrow \infty} P \times \operatorname{Leb}\left(A_{i}\right)=0$ and $\lim _{i \uparrow \infty} \mu^{m}\left(A_{i}\right)=0$. In addition, since $A_{i}^{c}=\cup_{n=1}^{i} S_{n}^{c}$, we have $\mu^{c h}\left(A_{i}^{c}\right) \leq \sum_{n=1}^{i} \mu^{c h}\left(S_{n}^{c}\right)=0$ and thus $\mu^{c h}\left(A_{i}^{c}\right)=0 \forall i$. Consider the auxilliary problems

$$
J_{i}=\sup _{c \in \mathcal{H}} \inf _{z \in b a \oplus, \lambda \in \mathrm{R}_{+}} U\left(1_{\left\{A_{i}^{c}\right\}} c\right)+\left\langle\mathcal{V}\left(1_{\left\{A_{i}^{c}\right\}} c\right), z\right\rangle+\mathcal{A}\left(1_{\left\{A_{i}^{c}\right\}} c\right) \lambda
$$

and let $\mathcal{O}$ be the set of predictable sets in $\Omega \times[0, T]$. Denote by $c^{i}, z^{i}$ and $\lambda^{i}$ the solutions of (85) for any $i$. Since $\mu^{c h}\left(A_{i}^{c}\right)=0 \forall i$, and $c$ is bounded, $\left\langle\mathcal{V}\left(1_{\left\{A_{i}^{c}\right\}} c\right), z^{i}\right\rangle=\int_{\Omega \times[0, T]} \mathcal{V}\left(1_{\left\{A_{i}^{c}\right\}} c\right) d \mu_{i}^{m}$ for some countably additive measure $\mu_{i}^{m}$ and $\forall c \in \mathcal{H}$. Define the nonnegative measure $\nu_{i}$ on $[0, T]$ as follows: $\left(P \times \nu_{i}\right)(E)=(P \times$ Leb $)(E) \forall E \in \mathcal{O}$ such that $(P \times$ Leb $)(E) \neq 0$ and $\left(P \times \nu_{i}\right)(E)=$ $\mu_{i}^{m}(E) \forall E \in \mathcal{O}$ such that $(P \times$ Leb $)(E)=0$. Since by construction $\mu_{i}^{m}$ is absolutely continuous with respect to $P \times \nu_{i}$, by the Radon-Nikodym theorem (i.e. Dunford and Schwartz (1958), III.10.2), one can write

$$
\left\langle\mathcal{V}\left(1_{\left\{A_{i}^{c}\right\}} c\right), z^{i}\right\rangle=\int \mathcal{V}\left(1_{\left\{A_{i}^{c}\right\}} c\right) d \mu_{i}^{m}=\int \mathcal{V}\left(1_{\left\{A_{i}^{c}\right\}} c\right) f^{i} d\left(P \times \nu_{i}\right)=E\left(\int_{0}^{T} \mathcal{V}_{t}\left(1_{\left\{A_{i}^{c}\right\}} c\right) f^{i} d \nu_{i}\right)
$$

for some nonnegative integrable function $f^{i}$. Defining the nonegative and nondecreasing process $\gamma_{t}^{i}=\int_{0}^{t} f_{s}^{i} d \nu_{i}$, we can then write $\left\langle\mathcal{V}\left(1_{\left\{A_{i}^{c}\right\}} c\right), z^{i}\right\rangle=E\left(\int_{0}^{T} \mathcal{V}_{t}\left(1_{\left\{A_{i}^{c}\right\}} c\right) d \gamma_{t}^{i}\right) \forall c \in \mathcal{H}$ 
By the constraint qualification condition (Luenberger (1969) Theorem 8.1) $\left\langle\mathcal{V}\left(1_{\left\{A_{i}^{c}\right\}} c^{i}\right), z^{i}\right\rangle$ $=0$ and $\mathcal{A}\left(1_{\left\{A_{i}^{c}\right\}} c\right) \lambda^{i}=0 \forall i$. Now, since all $c$ are bounded and the sequence $\left\{A_{i}^{c}\right\}$ is increasing, by monotone convergence we have $J_{i} \uparrow J$ and thus

$$
J=\sup _{c \in \mathcal{H}} \inf _{\gamma \in \mathcal{N}, \lambda \in \mathrm{R}_{+}} U(c)+E\left(\int_{0}^{T} \mathcal{V}_{t}(c) d \gamma_{t}\right)+\mathcal{A}(c) \lambda
$$

where $\mathcal{N}$ denotes the set of adapted, nonnegative and nondecreasing processes. Since

$$
E\left(\int_{0}^{T} \mathcal{V}_{t}(c) d \gamma_{t}\right)=E \int_{0}^{T} \int_{t}^{T} \xi_{s}\left(c_{s} \Leftrightarrow e_{s}\right) d s d \gamma_{t}
$$

integrating by parts and taking into account that $\gamma$ is nondecreasing and therefore of finite variation, we have (dropping the argument $c$ of $\mathcal{V}$ for convenience)

$$
0=\mathcal{V}_{T} \gamma_{T}=\int_{0}^{T} \mathcal{V}_{t} d \gamma_{t}+\int_{0}^{T} \gamma_{t} d \mathcal{V}_{t}+[\mathcal{V}, \gamma]_{T}=\int_{0}^{T} \mathcal{V}_{t} d \gamma_{t}+\int_{0}^{T} \gamma_{t} d \mathcal{V}_{t}
$$

write $\mathcal{V}_{t}=E\left(\int_{0}^{T} \xi_{s}\left(c_{s} \Leftrightarrow e_{s}\right) d s \mid \mathcal{F}_{t}\right) \Leftrightarrow \int_{0}^{t} \xi_{s}\left(c_{s} \Leftrightarrow e_{s}\right) d s$. Since the integrands are bounded, by the martingale representation theorem we can write $d \mathcal{V}_{t}=\Leftrightarrow\left(c_{t} \Leftrightarrow e_{t}\right) \xi_{t} d t+\phi_{t} d W_{t}$ where $\phi_{t}$ is an integrable adapted process. Substituting in (87) gives

$$
\int_{0}^{T} \mathcal{V}_{t} d \gamma_{t}=\Leftrightarrow \int_{0}^{T} \gamma_{t} \phi_{t} d W_{t}+\int_{0}^{T} \gamma_{t}\left(c_{t} \Leftrightarrow e_{t}\right) \xi_{t} d t
$$

and taking expectations, we obtain $E\left(\int_{0}^{T} \mathcal{V}_{t} d \gamma_{t}\right)=E\left(\int_{0}^{T} \gamma_{t}\left(c_{t} \Leftrightarrow e_{t}\right) \xi_{t} d t\right)$ so that (86) becomes

$$
J=\sup _{c} \inf _{\gamma, \lambda} U(c)+\lambda\left(X_{0} \Leftrightarrow E \int_{0}^{T} \xi_{t}\left(c_{t} \Leftrightarrow e_{t}\right) d t\right)+E\left(\int_{0}^{T} \gamma_{t}\left(c_{t} \Leftrightarrow e_{t}\right) \xi_{t} d t\right) .
$$

Taking the relevant (Gateaux) derivatives of (88), leads to the first order conditions (20).

\subsection{Appendix C: equilibrium with $\mathrm{N}$ agents.}

Consider the economy with $N^{u}$ unconstrained and $N^{c}$ constrained agents, $N^{u}+N^{c}=N$. To prove existence of equilibrium we need to solve

$$
\begin{gathered}
e_{t}=\sum_{j \in U} I_{j}\left(y^{j} \xi_{t}\right)+\sum_{j \in C} I_{j}\left(\left(y^{j} \Leftrightarrow \gamma_{t}^{j}\right) \xi_{t}\right) \\
\xi_{t} X_{t}^{j}=E\left(\int_{t}^{T} \xi_{s}\left[I_{j}\left(\left(y^{j} \Leftrightarrow \gamma_{s}^{j}\right) \xi_{s}\right) \Leftrightarrow e_{s}^{j}\right] d s \mid \mathcal{F}_{t}\right) \geq 0, \quad j \in C ; \\
X_{t}^{j} d \gamma_{t}^{j}=0, \gamma^{j} \text { nondecreasing null at } 0, \quad j \in C \\
E \int_{0}^{T} \xi_{s}\left[I_{j}\left(y^{j} \xi_{s}\right) \Leftrightarrow e_{s}^{j}\right] d s=n^{j} S_{0}, \quad j \in U \\
E \int_{0}^{T} \xi_{s}\left[I_{j}\left(\left(y^{j} \Leftrightarrow \gamma_{s}^{j}\right) \xi_{s}\right) \Leftrightarrow e_{s}^{j}\right] d s=n^{j} S_{0}, \quad j \in C
\end{gathered}
$$

Appealing to Walras law we can set the multiplier of one unconstrained agent, say agent 1 , equal to 1: $y^{1}=1$. Defining $y \equiv\left(y^{2}, \ldots, y^{N}\right)$ and $\gamma \equiv\left(\gamma^{2}, \ldots, \gamma^{N}\right)$ where $\gamma^{j} \equiv 0$ for $j \in U$ 
we obtain from the goods market clearing condition (89) $\xi_{t}=u_{c}\left(e_{t}, y \Leftrightarrow \gamma_{t}\right) \equiv g_{t}\left(y \Leftrightarrow \gamma_{t}\right)$ where $g_{t}(\cdot): \bigotimes_{j=2}^{N}\left[0, y^{j}\right] \rightarrow \mathbb{R}^{+}$is the unique solution of $e_{t}=\sum_{j \in U} I_{j}\left(y^{j} g_{t}\left(y \Leftrightarrow \gamma_{t}\right)\right)+\sum_{j \in C} I_{j}\left(\left(y^{j} \Leftrightarrow\right.\right.$ $\left.\left.\gamma_{t}^{j}\right) g_{t}\left(y \Leftrightarrow \gamma_{t}\right)\right)$. Substituting in (90)-(93) and defining the process $h_{t}^{j}(\cdot)$

$$
h_{t}^{j}(x)=g_{t}(x)\left[I_{j}\left(x^{j} g_{t}(x)\right) \Leftrightarrow e_{t}^{j}\right]
$$

with the convention $x^{j}=y^{j}$ for $j \in U$, leaves us with the system

$$
\begin{aligned}
& \xi_{t} X_{t}^{j}=E\left(\int_{t}^{T} h_{s}^{j}\left(y \Leftrightarrow \gamma_{s}\right) d s \mid \mathcal{F}_{t}\right) \geq 0, \quad j \in C \\
& X_{t}^{j} d \gamma_{t}^{j}=0, \gamma^{j} \text { nondecreasing null at } 0, \quad j \in C \\
& E \int_{0}^{T} h_{s}^{j}\left(y \Leftrightarrow \gamma_{s}\right) d s=n^{j} S_{0}, \quad j \in C \cup U \backslash 1 .
\end{aligned}
$$

The functions $g$ and $h$ have the following properties

Lemma 21 For all $t \in[0, T]$ the function $g_{t}(\cdot): \bigotimes_{j=2}^{N}\left[0, y^{j}\right] \rightarrow\left[g_{t}(y), \infty\right)$ satisfies

(i) $g_{t}(\cdot)$ is strictly decreasing with respect to each argument $x^{j}$

(ii) $\lim _{x^{j} \rightarrow 0} g_{t}(x)=\infty$

(iii) $\lim _{x \rightarrow y} g_{t}(x)=g_{t}(y)>0$

(iv) $\frac{\partial g_{t}(x)}{\partial x^{j}}=\Leftrightarrow \frac{I_{j}^{\prime}\left(x^{j} g_{t}(x)\right) g_{t}(x)}{I_{1}^{\prime}\left(g_{t}(x)\right)+\sum_{i \neq 1} I_{i}^{\prime}\left(x^{i} g_{t}(x)\right) x^{i}}$.

Proof of Lemma 21: The function $g_{t}(\cdot)$ is continuously differentiable over its domain. An application of the Implicit Function Theorem yields (iv). Property (i) follows from (iv). When $x^{j} \rightarrow 0$ we have $I_{j}\left(x^{j} g_{t}(x)\right) \rightarrow \infty$ for all $g_{t}(x)$ finite. Since the left hand side of (89) is finite and $I_{j}(\cdot)$ is nonnegative for all $j$ the limit (ii) holds. Property (iii) is straightforward.

Lemma 22 For all $t \in[0, T]$ the function $h_{t}^{j}(\cdot): \bigotimes_{j=2}^{N}\left[0, y^{j}\right] \rightarrow\left[h_{t}^{j}(y), 0\right]$ satisfies

(i) $\exists x^{j *} \in\left(0, y^{j}\right)$ such that $h_{t}^{j}(x)$ is strictly decreasing for $x^{j}<x^{j *}$

(ii) $\lim _{x^{j} \rightarrow 0} h_{t}^{j}(x)=\infty$

(iii) $\lim _{x \rightarrow y} h_{t}^{j}(x)=h_{t}^{j}(y)>(<) 0 \Leftrightarrow I_{j}\left(y^{j} g_{t}(y)\right) \Leftrightarrow e_{s}^{j}>(<) 0$.

(iv) $\frac{\partial h_{t}^{j}(x)}{\partial x^{j}}=\frac{\partial g_{t}(x)}{\partial x^{j}}\left[I_{j}\left(x^{j} g_{t}(x)\right) \Leftrightarrow e_{t}^{j}\right]+g_{t}(x) I_{j}^{\prime}\left(x^{j} g_{t}(x)\right)\left[g_{t}(x)+x^{j} \frac{\partial g_{t}(x)}{\partial x^{j}}\right]$.

Proof of Lemma 22: Properties (ii), (iii) and (iv) are straightforward. To prove (i) note that (89) and property (ii) of lemma 21 imply $I_{j}\left(x^{j} g_{t}(x)\right) \Leftrightarrow e_{t}^{j} \geq 0$ for $x^{j}$ sufficiently low. Let $x^{j *}$ be such that $I_{j}\left(x^{j *} g_{t}\left(x^{*}\right)\right) \Leftrightarrow e_{t}^{j}=0$. Property (iv) of Lemma 21 implies

$$
g_{t}(x)+x^{j} \frac{\partial g_{t}(x)}{\partial x^{j}}=\frac{\left[I_{1}^{\prime}\left(g_{t}(x)\right)+\sum_{i \neq 1, j} I_{i}^{\prime}\left(x^{i} g_{t}(x)\right) x^{i}\right] g_{t}(x)}{I_{1}^{\prime}\left(g_{t}(x)\right)+\sum_{i \neq 1} I_{i}^{\prime}\left(x^{i} g_{t}(x)\right) x^{i}}>0 .
$$

Hence $I_{j}\left(x^{j} g_{t}(x)\right) \Leftrightarrow e_{t}^{j}$ is decreasing and for $x^{j}<x^{j *}$ we have $I_{j}\left(x^{j} g_{t}(x)\right) \Leftrightarrow e_{t}^{j} \geq 0$. Since $\partial g_{t}(x) / \partial x^{j}<0$ it then follows from (iv) that $\partial h_{t}^{j}(x) / \partial x^{j}<0$ for $x^{j}<x^{j *}$.

Consider now the $N^{c}$-dimensional vector process

$$
F_{t}\left(y, \Delta_{t}\right)=E\left(\int_{t}^{T} h_{s}\left(y \Leftrightarrow \Delta_{t, s}^{*}\right) d s \mid \mathcal{F}_{t}\right), \quad t \in[0, T]
$$

where $\Delta_{t, s}^{*}=\sup _{v \in[t, s]} \Delta_{v}$. We need to solve the auxiliary problem, for all $t \in[0, T]$ 


$$
\begin{aligned}
F_{t}\left(y, \Delta_{t}\right) & \geq 0 \\
F_{t}\left(y, \Delta_{t}\right) * d \Delta_{t} & \leq 0
\end{aligned}
$$

where * denotes multiplication component by component of two vectors. Label the $N^{c}$ components of (99)-(100) by $j=1, \ldots, N^{c}$. Consider the first component of (99)-(100). The problem of solving for $\Delta^{1}$ taking $y$ and $\Delta^{i}, i \neq 1$ as given is identical to the problem that we solved before for the case of 2 agents. By Lemma 22 the integrand $h_{t}^{1}(x)$ has the required properties. Hence

$$
\Delta^{1}\left(y, \Delta^{-(1)}\right)=F_{t}^{-1}\left(y, 0, \Delta^{-(1)}\right) 1_{\left\{F_{t}\left(y, 0, \Delta_{t}^{-(1)}\right)<0\right\}}
$$

where $\Delta^{-(1)} \equiv\left(\Delta^{2}, \ldots, \Delta^{N^{c}}\right)$. Consider next the second component of (99)-(100) where $\Delta^{1}\left(y, \Delta^{-(1)}\right)$ has been substituted in place of $\Delta^{1}$ and where $y$ and $\Delta^{i}, i \neq 1,2$ are taken as given. If the same construction can be carried out we can define a new process $\Delta^{2}\left(y, \Delta^{-(1,2)}\right)$ where $\Delta^{-(1,2)} \equiv\left(\Delta^{3}, \ldots, \Delta^{N^{c}}\right)$. Substituting in the first process yields

$$
\Delta^{1}\left(y, \Delta^{-(1,2)}\right) \equiv \Delta^{1}\left(y, \Delta^{2}\left(y, \Delta^{-(1,2)}\right), \Delta^{-(1,2)}\right) .
$$

Proceeding inductively over $j=1, \ldots, N^{c}$, we can, in principle, construct the array of processes

$$
\Delta^{(j)}\left(y, \Delta^{-(1, . ., j)}\right) \equiv\left(\Delta^{1}\left(y, \Delta^{-(1, . ., j)}\right), \ldots, \Delta^{j}\left(y, \Delta^{-(1, . ., j)}\right)\right)
$$

where $\Delta^{-(1, . ., j)} \equiv\left(\Delta^{j+1}, \ldots, \Delta^{N^{c}}\right)$ for $j=1, \ldots, N^{c}$. Our next lemma establishes the validity of this construction.

Lemma 23 Suppose that $\Delta^{(j-1)}\left(y, \Delta^{-(1, . ., j-1)}\right)$ exists, for some $j=1, \ldots, N^{c}$. Then $\Delta^{(j)}\left(y, \Delta^{-(1, . ., j)}\right)$ exists.

Proof of Lemma 23: To simplify notation define

$$
\left\{\begin{array}{c}
x^{i}\left(x^{-(1, . ., j-1)}\right) \equiv y^{i} \Leftrightarrow \Delta^{i}\left(y, \Delta^{-(1, . ., j-1)}\right), \quad \text { for } i=1, \ldots, j \Leftrightarrow 1 \\
x^{(j-1)}\left(x^{-(1, . ., j-1)}\right) \equiv\left(x^{1}\left(x^{-(1, . ., j-1)}\right), \ldots, x^{j-1}\left(x^{-(1, . ., j-1)}\right)\right)=y^{(j-1)} \Leftrightarrow \Delta^{(j-1)}\left(y, \Delta^{-(1, . ., j-1)}\right)
\end{array}\right.
$$

where $x^{-(1, . ., j-1)}=\left(x^{j}, \ldots, x^{\left.N^{c}\right)}\right)$ is taken as given and consider the map

$$
h^{j}(x) \equiv h^{j}\left(x^{1}\left(x^{-(1, . ., j-1)}\right), \ldots, x^{j-1}\left(x^{-(1, . ., j-1)}\right), x^{j}, \ldots, x^{N^{c}}\right)
$$

Suppose that $x_{t}^{j} \rightarrow 0$. Since $x^{j}$ is a nonincreasing process we must have $x_{v}^{j} \rightarrow 0$ for all $v \in[t, T]$. Property (ii) of Lemma 21 then implies that $g_{v}(x) \rightarrow \infty$ for all $v \in[t, T]$. Furthermore since the first $j \Leftrightarrow 1$ conditions of (99)-(100) hold by definition of the maps $x^{i}\left(x^{-(1, . ., j-1)}\right), i=1, \ldots, j \Leftrightarrow 1$ it must also be that

$$
x_{v}^{1}\left(y, x^{-(1, . ., j-1)}\right) \rightarrow 0, . ., x_{v}^{j-1}\left(y, x^{-(1, . ., j-1)}\right) \rightarrow 0, \quad \text { for all } v \in[t, T]
$$

since otherwise $h_{v}^{j}\left(x^{j}\right) \rightarrow \Leftrightarrow \infty$, for all $v \in[t, T]$. In fact $F_{v}^{i} \rightarrow 0$ for all $v \in[t, T]$ and $i=1, \ldots, j \Leftrightarrow 1$, i.e. the constraints must be binding, on the first $j \Leftrightarrow 1$ agents and

$$
I\left(x_{t}^{i}\left(y, x^{-(1, . ., j-1)}\right) g_{t}(x)\right) \Leftrightarrow e_{t}^{i} \rightarrow 0, \text { for all } i=1, \ldots, j \Leftrightarrow 1 .
$$

The market clearing condition (89) then implies $I\left(x_{v}^{j} g_{v}(x)\right) \Leftrightarrow e_{v}^{j} \geq 0$. Thus $h^{j} \rightarrow \infty$. Continuity of $h^{j}$ implies that there exists an $x^{j}$ such that $F_{t}^{j} \geq 0$. The existence of $\Delta^{(j)}\left(y, \Delta^{-(1, \ldots, j)}\right)$ follows.

An immediate consequence of the previous lemma is the following result. 
Theorem 24 For each $y>0$ the system (95)-(96) has a solution $\gamma(y)=\left(\gamma^{1}(y), \ldots, \gamma^{N^{c}}(y)\right)$.

Proof of Theorem 24: Lemma 23 and the existence of $\Delta^{1}\left(y, \Delta^{-(1)}\right)$ implies that we can construct a vector process $\Delta(y)$ which satisfies (99)-(100). The process $\gamma(y)$ is $\gamma_{t}(y)=\Delta_{0, t}^{*}(y)$.

Lemma 25 There exists $y^{*}=\left(y^{* 2}, \ldots, y^{* N}\right) \in(0, \infty)^{N-1}$ such that

$$
E \int_{0}^{T} h_{s}^{j}\left(y^{*} \Leftrightarrow \gamma_{s}\left(y^{*}\right)\right) d s=n^{j} S_{0}, \quad j \in C \cup U \backslash 1
$$

where $\gamma(y)=\gamma^{(N)}(y)$.

Proof of Lemma 25: This follows from the Knaster-Kuratowski-Mazurkiewicz Theorem. See Karatzas, Lakner, Lehoczky and Shreve (1991).

Summarizing we have proved the following result

Theorem 26 The system (95)-(97) has a solution $\gamma^{*}=\left(\gamma^{1}\left(y^{*}\right), \ldots, \gamma^{N}\left(y^{*}\right)\right)$ where $\gamma_{t}(y)=\Delta_{0, t}^{*}(y)$ solves (95)-(96) and $y^{*}$ is the solution of (102).

\subsection{Appendix D: computation based on a binomial approximation.}

We consider now a simple binomial model with underlying underlying uncertainty process $z$ and parameters $u$ and $d$, and probability $p$.

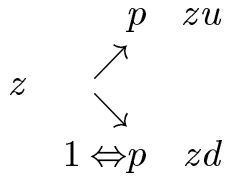

The initial value is $z_{0}$ and the tree has $N$ steps. Agents endowments are functionals of the trajectories of the random walk $z$. Since prices and the interest rate are endogenous the risk neutral probability is given by some measure $q \equiv\left\{q_{n}: n=1, \ldots, N\right\}$ and the interest rate by some process $r$. The state price density satisfies

$$
r_{n} \xi_{n+1}=\xi_{n}\left\{\begin{array}{c}
q_{n} / p \\
\left(1 \Leftrightarrow q_{n}\right) /(1 \Leftrightarrow p)
\end{array} .\right.
$$

Equilibrium in the 2-agents economy is the solution of

$$
\begin{gathered}
\xi_{n} X_{n}^{2}=E\left(\sum_{i=n}^{N} h_{i}\left(y \Leftrightarrow \gamma_{i}\right) \mid \mathcal{F}_{n}\right) \geq 0, \quad n \in\{1, \ldots, N\} \\
X_{n}^{2} \Delta \gamma_{n}=0, \gamma \text { nondecreasing null at } 0, \quad n \in\{1, \ldots, N\} \\
E \sum_{i=0}^{N} h_{i}\left(y \Leftrightarrow \gamma_{i}\right)=0
\end{gathered}
$$

where $g$ is constructed from the aggregate demand and $h_{n}(x)=g_{n}(x)\left[I_{2}\left(x g_{n}(x)\right) \Leftrightarrow e_{n}^{2}\right]$. In order to solve for equilibrium we introduce an auxiliary process $\psi$ and the map

$$
A_{n}\left(y, \psi_{n}\right) \equiv E\left(\sum_{i=n+1}^{N} h_{i}\left(y \Leftrightarrow \max \left(\psi_{i}, \ldots, \psi_{n}\right)\right) \mid \mathcal{F}_{n}\right)
$$


Consider now the backward equation

$$
\psi_{n}=\left\{\begin{array}{c}
0 \quad \text { if } h_{n}(y)+A_{n}(y, 0) \geq 0 \\
\max \left\{y \Leftrightarrow h_{n}^{-1}\left(\Leftrightarrow A_{n}\left(y, \psi_{n}\right)\right), 0\right\} \quad \text { if } h_{n}(y)+A_{n}(y, 0)<0
\end{array}\right.
$$

for $n=1, \ldots, N$ subject to the boundary condition

$$
\psi_{N}=\left\{\begin{array}{c}
0 \quad \text { if } h_{N}(y) \geq 0 \\
y \Leftrightarrow h_{N}^{-1}(0) \quad \text { if } h_{N}(y)<0
\end{array}\right.
$$

In these equations $h_{n}^{-1}(x)$ is the largest value of the argument of $h_{n}$ that reaches the level $x$. It is easy to verify that the process $\gamma$ can be recovered from the solution of the backward equation as $\gamma_{n}=\max \left(\psi_{n}, \ldots, \psi_{1}\right)$. Our next lemma establishes the existence of the process $\psi$.

Lemma 27 The backward equation (107)-(108) has a unique solution $\psi_{n}(y)$.

Proof of Lemma 27: Suppose that $h_{n}(y)+A_{n}(y, 0)<0$. Then the recursive equation $\psi_{n}=$ $y \Leftrightarrow h_{n}^{-1}\left(\Leftrightarrow A_{n}\left(y, \psi_{n}\right)\right)$ is equivalent to $h_{n}\left(y \Leftrightarrow \psi_{n}\right)+A_{n}\left(\psi_{n}\right)=0$.As in appendix A it can be verified that the left hand side of this equation is strictly increasing with respect to $\psi_{n}$ for $y \Leftrightarrow \psi_{n}$ sufficiently small and that it converges to infinity as $y \Leftrightarrow \psi_{n}$ goes to zero. The result follows by continuity.

The process $\gamma$ is then given by $\gamma_{n}(y)=\max \left(\psi_{n}(y), \ldots, \psi_{1}(y)\right)$, where $\gamma(y)$ denotes the solution for a given constant $y$. We have

Lemma 28 The map $\gamma(y)$ has the following properties (i) $\gamma(y)$ is a nondecreasing function of $y$, (ii) $\lim _{y \rightarrow 0} \gamma(0)=0$, (iii) $\lim _{y \rightarrow \infty} \gamma(y)=\infty$, (iv) $\lim _{y \rightarrow \infty} y \Leftrightarrow \gamma(y) \geq 0$.

Lemma 29 There exists a multiplier $y^{*}$ such that $h_{0}\left(y^{*}\right)+A_{0}\left(y^{*}, 0\right)=0$. The nondecreasing multiplier $\gamma\left(y^{*}\right)$ is $\gamma_{n}\left(y^{*}\right)=\max \left(\psi_{n}\left(y^{*}\right), \ldots, \psi_{1}\left(y^{*}\right)\right)$.

Proof of Lemma 29: The multiplier for the date zero budget constraint satisfies

$$
h_{0}(y)+E\left(\sum_{i=1}^{N} h_{i}\left(y \Leftrightarrow \gamma_{i}(y)\right)\right)=0 .
$$

Using Lemma 28 above and the properties of the map $h$ it can be verified that a solution exists

The equilibrium state price density is then $\xi_{n}=g_{n}\left(y \Leftrightarrow \gamma_{n}(y)\right)$. It generates the equations

$$
\left\{\begin{array}{c}
r_{n} \frac{g_{n+1}^{u}\left(y-\gamma_{n+1}(y)\right)}{g_{n}\left(y-\gamma_{n}(y)\right)} p=q_{n} \\
r_{n} \frac{g_{n+1}^{d}\left(y-\gamma_{n+1}(y)\right)}{g_{n}\left(y-\gamma_{n}(y)\right)}(1 \Leftrightarrow p)=1 \Leftrightarrow q_{n} .
\end{array}\right.
$$

Solving this system for $r$ and $q$ yields

$$
\begin{gathered}
q_{n}=g_{n+1}^{u}\left(y \Leftrightarrow \gamma_{n+1}(y)\right) p\left[g_{n+1}^{d}\left(y \Leftrightarrow \gamma_{n+1}(y)\right)+\left[g_{n+1}^{u}\left(y \Leftrightarrow \gamma_{n+1}(y)\right) \Leftrightarrow g_{n+1}^{d}\left(y \Leftrightarrow \gamma_{n+1}(y)\right)\right] p\right]^{-1} \\
r_{n}=g_{n}\left(y \Leftrightarrow \gamma_{n}(y)\right)\left[g_{n+1}^{d}\left(y \Leftrightarrow \gamma_{n+1}(y)\right)+\left[g_{n+1}^{u}\left(y \Leftrightarrow \gamma_{n+1}(y)\right) \Leftrightarrow g_{n+1}^{d}\left(y \Leftrightarrow \gamma_{n+1}(y)\right)\right] p\right]^{-1} .
\end{gathered}
$$

Using the liquid wealth process and defining $V_{n}^{j}=g_{n}\left(y \Leftrightarrow \gamma_{n}(y)\right)^{-1} E_{n}\left[\sum_{i=n}^{N} g_{i}\left(y \Leftrightarrow \gamma_{i}(y)\right) e_{i}^{j}\right]$ we can now write 


$$
\begin{aligned}
& r_{n} X_{n}^{j}+\pi_{n}^{j}\left(u_{n} \Leftrightarrow r_{n}\right) \Leftrightarrow c_{n}^{j}+e_{n}^{j}+r_{n} V_{n}^{j}+\left(1 \Leftrightarrow q_{n}\right)\left(V_{n+1}^{j u} \Leftrightarrow V_{n+1}^{j d}\right) \Leftrightarrow e_{n}^{j} \\
= & \left(\xi_{n+1}^{u}\right)^{-1}\left\{G_{n+1}^{j} \Leftrightarrow \sum_{i=0}^{n} g_{i}\left(y \Leftrightarrow \gamma_{i}(y)\right) I_{j}\left(\left(y \Leftrightarrow \gamma_{i}(y)\right) g_{i}\left(y \Leftrightarrow \gamma_{i}(y)\right)\right)\right\}
\end{aligned}
$$

and a similar equation for the down step. Using $c_{n}^{j}=I_{j}\left(y \Leftrightarrow \gamma_{n}\right)$ and

$$
r_{n} \xi_{n+1}^{u}=\xi_{n} \cdot\left(q_{n} / p\right)=\left(X_{n}^{j}+V_{n}^{j}\right)^{-1}\left[G_{n}^{j} \Leftrightarrow \sum_{i=0}^{n-1} g_{i}\left(y \Leftrightarrow \gamma_{i}(y)\right) I_{j}\left(\left(y \Leftrightarrow \gamma_{i}(y)\right) g_{i}\left(y \Leftrightarrow \gamma_{i}(y)\right)\right)\right]\left(q_{n} / p\right)
$$

we then get the optimal portfolio

$$
\pi_{n}^{j}=\left(X_{n}^{j}+V_{n}^{j}\right) \frac{r_{n}}{u_{n} \Leftrightarrow r_{n}}\left[J_{n, n+1}^{j} \frac{p}{q_{n}} \Leftrightarrow 1\right]+\frac{I_{j}\left(y \Leftrightarrow \gamma_{n}(y)\right)}{u_{n} \Leftrightarrow r_{n}} \Leftrightarrow \frac{\left(1 \Leftrightarrow q_{n}\right)\left(V_{n+1}^{j u} \Leftrightarrow V_{n+1}^{j d}\right)}{u_{n} \Leftrightarrow r_{n}}
$$

where $V_{n}^{j}$ is defined above and

$$
J_{n, n+1}^{j}=\frac{\left[G_{n+1}^{j} \Leftrightarrow \sum_{i=0}^{n} g_{i}\left(y \Leftrightarrow \gamma_{i}(y)\right) I_{j}\left(\left(y \Leftrightarrow \gamma_{i}(y)\right) g_{i}\left(y \Leftrightarrow \gamma_{i}(y)\right)\right)\right]}{\left[G_{n}^{j} \Leftrightarrow \sum_{i=0}^{n-1} g_{i}\left(y \Leftrightarrow \gamma_{i}(y)\right) I_{j}\left(\left(y \Leftrightarrow \gamma_{i}(y)\right) g_{i}\left(y \Leftrightarrow \gamma_{i}(y)\right)\right)\right]} .
$$




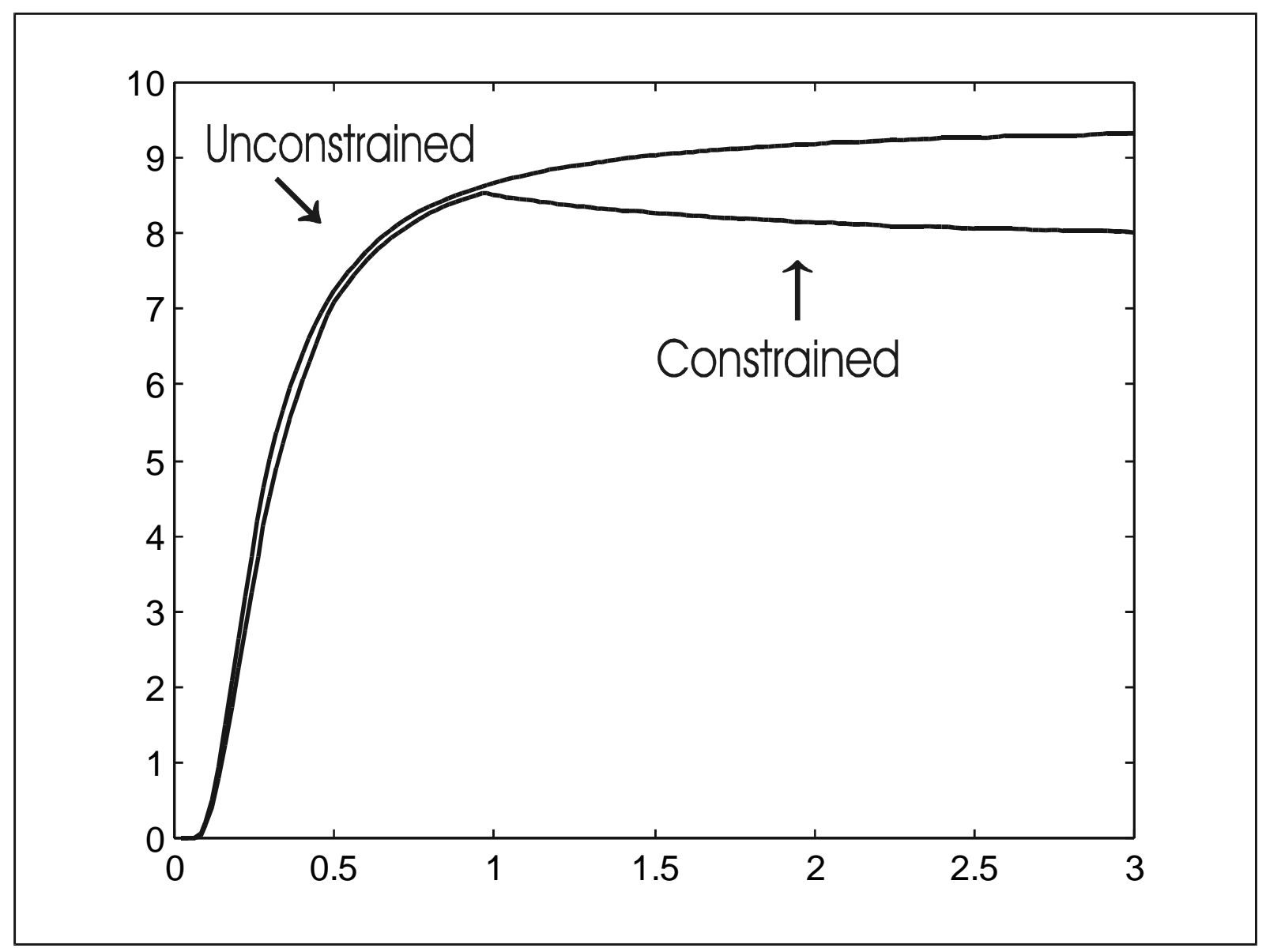

Figure 1 


\section{Liste des publications au CIRANO *}

\section{Cahiers CIRANO / CIRANO Papers (ISSN 1198-8169)}

96c-1 Peut-on créer des emplois en réglementant le temps de travail ? / Robert Lacroix

95c-2 Anomalies de marché et sélection des titres au Canada / Richard Guay, Jean-François L'Her et Jean-Marc Suret

95c-1 La réglementation incitative / Marcel Boyer

94c-3 L'importance relative des gouvernements : causes, conséquences et organisations alternative / Claude Montmarquette

94c-2 Commercial Bankruptcy and Financial Reorganization in Canada / Jocelyn Martel

94c-1 Faire ou faire faire : La perspective de l'économie des organisations / Michel Patry

\section{Série Scientifique / Scientific Series (ISSN 1198-8177)}

98s-40 Monetary Policy Rules with Model and Data Uncertainty / Eric Ghysels, Norman R. Swanson et Myles Callan

98s-39 Environmental Risks: Should Banks Be Liable? / Karine Gobert et Michel Poitevin

98s-38 Better Observability Promotes the Adoption of More Flexible Technologies / Marcel Boyer, Armel Jacques et Michel Moreaux

98s-37 Job Characteristics, Wages and the Employment Contract / W. Bentley MacLeod et Daniel Parent

98s-36 Observability, Commitment and Flexibility / Marcel Boyer, Armel Jaques et Michel Moreaux

98s-35 Pricing ang Hedging Derivative Securities with Neural Networks and a Homogeneity Hint / René Garcia et Ramazan Gençay

98s-34 Gender Composition and Wages: Why Is Canada Different from the United States? / Michael Baker et Nicole M. Fortin

98s-33 Effects of the Fiscal Treatment of Tax Losses on the Efficiency of Markets and the Incidence of Mergers / Michel Poitevin

98s-32 Travail pendant les études et abandon scolaire : Causes, conséquences, et politiques d'intervention / Marcel Dagenais, Claude Montmarquette, Daniel Parent, Benoit Durocher et François Raymond

98s-31 Collusive Allocation of Tradeable Pollution permits / Ngo Van Long et Antoine Soubeyran

98s-30 Pollution, Pigouvian Taxes, and Asymmetric International Oligopoly / Ngo Van Long et Antoine Soubeyran

98s-29 Quadratic M-Estimators for ARCH-Type Processes / Nour Meddahi et Éric Renault

98s-28 Explaining Sales Pay Strategy Using Agency, Transaction Cost and Resource Dependence Theories / Michel Tremblay, Jérôme Côté et David B. Balkin

98s-27 The Moderating Effect of Job Characteristics on Managers' Reactions to Career Plateau / Michel Tremblay et Alain Roger

* Vous pouvez consulter la liste complète des publications du CIRANO et les publications elles-mêmes sur notre site World Wide Web à l'adresse suivante :

http://www.cirano.umontreal.ca/publication/page1.html 